\title{
Ontogeny of Modulatory Inputs to Motor Networks: Early Established Projection and Progressive Neurotransmitter Acquisition
}

\author{
Yves Le Feuvre, Valérie S. Fénelon, and Pierre Meyrand \\ Laboratoire de Neurobiologie des Réseaux, Centre National de la Recherche Scientifique Unité Mixte de Recherche 5816, \\ Université Bordeaux I, 33405 Talence cedex, France
}

\begin{abstract}
Modulatory information plays a key role in the expression and the ontogeny of motor networks. Many developmental studies suggest that the acquisition of adult properties by immature networks involves their progressive innervation by modulatory input neurons. Using the stomatogastric nervous system of the European lobster Homarus gammarus, we show that contrary to this assumption, the known population of projection neurons to motor networks, as revealed by retrograde dye migration, is established early in embryonic development. Moreover, these neurons display a large heterogeneity in the chronology of acquisition of their full adult neurotransmitter phenotype.

We performed retrograde dye migration to compare the neuronal population projecting to motor networks located in the stomatogastric ganglion in the embryo and adult. We show that this neuronal population is quantitatively established at developmental stage $65 \%$, and each identified projection neuron
\end{abstract}

displays the same axon projection pattern in the adult and the embryo. We then combined retrograde dye migration with FLRFamide-like, histamine, and GABA immunocytochemistry to characterize the chronology of neurotransmitter expression in individual identified projection neurons. We show that this early established population of projection neurons gradually acquires its neurotransmitter phenotype complement. This study indicates that (1) the basic architecture of the known population of projection inputs to a target network is established early in development and (2) ontogenetic plasticity may depend on changes in neurotransmitter phenotype expression within preexisting neurons rather than in the addition of new projection neurons or fibers.

Key words: central pattern generator; development; modulatory neurons; FLRFamide; GABA; histamine
Modulatory processes play a crucial role in the expression of neural networks in the adult CNS. Modulatory neurons both initiate short-term changes in the activity of a given network according to environmental needs (Marder and Calabrese, 1996) and exert long-lasting effects that maintain network integrity (Thoby-Brisson and Simmers, 1998; McKinney et al., 1999). Neuromodulatory systems involved in the control of central pattern generators (CPGs) have been extensively studied in terms of neuronal population, neurotransmitter phenotype, and alteration of network activity. A large body of work has been devoted to the progressive developmental acquisition of projection neurons using retrograde dye migration (Cabana and Martin, 1984; van Mier and ten Donkelaar, 1984; Okado and Oppenheim, 1985; Kudo et al., 1993) and of neuromodulatory substances using immunocytochemistry (Senba et al., 1982; Commissiong, 1983; Sako et al., 1986; van Mier et al., 1986; Rajaofetra et al., 1989; Pindzola et al., 1990; Fenelon et al., 1998a, 1999; Kilman et al., 1999). Using these approaches, it has been concluded that progressive acquisition of neuromodulatory substances by a target network is

\footnotetext{
Received Aug. 1, 2000; revised Nov. 20, 2000; accepted Nov. 28, 2000.

This work was supported by a doctoral student fellowship from Ministère de la Recherche et de la Technologie to Y.L. and the Conseil Régional d'Aquitaine. We thank Lionel Para-iglesias and Philippe Chauvet for setting up the animal facility and taking great care of the juvenile, adult, and egg-bearing females that were used for this study. We deeply thank Dr. R. Miles for reviewing an earlier version of this manuscript.

Correspondence should be addressed to Yves Le Feuvre, Laboratoire de Neurobiologie des Réseaux, Centre National de la Recherche Scientifique Unité Mixte de Recherche 5816, Université Bordeaux I, Biologie Animale Bâtiment B2, Avenue des Facultés, 33405 Talence cedex, France. E-mail: y.lefeuvre@Inr.u-bordeaux.fr. Copyright (C) 2001 Society for Neuroscience $0270-6474 / 01 / 211313-14 \$ 15.00 / 0$
}

associated with axonal ingrowth of descending neurons (Sako et al., 1986; van Mier et al., 1986; Pindzola et al., 1990). However, some studies seem to indicate that projection neurons may reach their targets before expressing their adult neurotransmitter complement (Henderson, 1991; Kilman et al., 1999). To investigate whether such ontogenetic processes may exist in descending systems, we combined retrograde dye migration to label embryonic neurons reaching their target networks, and immunocytochemical detection of neuromodulatory substances.

A suitable preparation for such study is the stomatogastric nervous system (STNS) of the lobster. In this model, two neuronal networks, located in the stomatogastric ganglion (STG), generate two motor outputs controlled by identified central and peripheral modulatory neurons (Katz et al., 1989; Meyrand et al., 1991, 1994, 2000; Nagy et al., 1994; Combes et al., 1999). Moreover, central projection neurons play a key role in the ontogeny of STNS networks because their presence masks preexisting adultlike phenotypes (Le Feuvre et al., 1999). In addition, immunocytochemical characterization of these inputs has shown that modulatory substances appear gradually in the STG during embryonic development (Cournil et al., 1995; Fenelon et al., 1998a, 1999; Kilman et al., 1999). However, such results do not exclude that some projection neurons may reach their target networks without expressing their final neurotransmitter phenotype. We therefore investigated whether neuromodulator phenotypes are progressively acquired within an already established set of projection neurons or within newly formed projection neurons.

Our data suggest that (1) all known adult projection neurons to the STG are present and reach their target network in the second 
half of the embryonic life, (2) each projection neuron displays its own developmental chronology of neurotransmitter appearance, and (3) the time of acquisition of a given neuromodulatory substance can differ from one neuron to another.

Together, these data indicate that ontogenetic plasticity expressed by neuromodulatory systems may depend on alteration of neurotransmitter phenotype expression within preexisting neurons rather than the addition of new projection fibers.

\section{MATERIALS AND METHODS}

Animals and dissection. Experiments were performed on embryos, juveniles, and adults of the European lobster Homarus gammarus. Male adults and egg-bearing females were obtained from a local fishery supply, and juveniles were purchased from the South Wexford Lobster Coop (South Wexford, Ireland). Animals were kept in large tanks of $15^{\circ} \mathrm{C}$ aerated circulating artificial seawater. The percentage staging system based on eye index was used to determine the age of the embryos (Helluy and Beltz, 1991). All embryonic stages used in this study ranged from 60 to $95 \%$ development, so that the preparation had a sufficient size to perform dye migration. Juvenile animals used for the experiments had cephalothorax lengths (from the anterior point of the rostrum to the posterior edge of the thorax) ranging from 8.5 to $10 \mathrm{~mm}$. Experiments on the adult were performed on male animals weighing $300 \mathrm{gm}$.

Dissections were performed in aerated physiological saline containing (in mM): $\mathrm{NaCl} 479.12, \mathrm{KCl} 12.74, \mathrm{CaCl}_{2} 13.2, \mathrm{MgSO}_{4} 10, \mathrm{Na}_{2} \mathrm{SO}_{4} 3.2$, and HEPES 5, pH 7.45. Adult (see Fig. $1 A$ ) and embryonic (see Fig. $1 B$ ) STNSs were dissected as described previously (Casasnovas and Meyrand, 1995). Briefly, for the embryo, the thin membranes protecting the embryo were removed, and the stomodeum was isolated with the brain and the anterior part of the ventral nerve cord. The stomach and the ventral nerve cord were then split open along the ventral midline and pinned on a Sylgard-coated Petri dish. To access the main nerves of the embryonic STNS, anterior ganglia and related nerves were dissected using small tungsten pins, and the muscular part of the esophagus was removed (see Fig. 1B2).

Retrograde labeling of projection neurons. To label the neurons with axons projecting via a given nerve, a small Vaseline well was built around the nerve before it was cut. The saline in this well was replaced by distilled water, and the nerve was then cut. After 5 min the water was replaced with 5\% dextran tetramethyl rhodamine (Molecular Probes, Eugene, OR) [3000 molecular weight (MW)] in $0.2 \mathrm{M}$ potassium acetate and left for $1-2 \mathrm{hr}$ at $13^{\circ} \mathrm{C}$ for embryos, or $12 \mathrm{hr}$ to $2 \mathrm{~d}$ at $4^{\circ} \mathrm{C}$ for juveniles and adults. The dye and Vaseline were removed, and the preparation was rinsed with fresh saline. The stained neurons were visualized in toto using a laser scanning confocal microscope (Leica TCS 4D).

Immunocytochemistry. To characterize the neuromodulators expressed by a given projection neuron, double stainings were performed. First, back fills were performed as above. The dissected adult, juvenile, and embryonic STNSs, while pinned on their Sylgard-coated Petri dishes, were processed for immunolabeling of GABA, histamine, and FLRFamide-like peptides using indirect immunofluorescent techniques. We used a polyclonal serum raised in rabbit against GABA (Sigma, St. Louis, MO) at a dilution of 1:200. The extended FLRFamide-like peptides (Trimmer et al., 1987) were detected with a 1:800 dilution of a polyclonal antiserum (Diasorin, Stillwater, $\mathrm{MN}$ ) raised in rabbit against FMRFamide [for specificity see Fenelon et al. (1998a)]. Finally, histamine immunoprocessing used a rabbit polyclonal antibody (Accurate Chemical and Scientific Corporation) at a final dilution of 1:1000. This antibody was a kind gift from Dr. M. P. Nusbaum (University of Pennsylvania, Philadelphia, PA). For GABA and FLRFamide detection, preparations were fixed with $4 \%$ paraformaldehyde in $0.1 \mathrm{M} \mathrm{PBS,} \mathrm{pH} \mathrm{7.4,}$ $115.5 \mathrm{~mm} \mathrm{NaCl}$, and $4 \mathrm{~mm} \mathrm{KCl}$ solution for 1 or $12 \mathrm{hr}$, respectively. To detect histamine immunoreactivity, preparations were dissected in a modified low calcium saline containing (in $\mathrm{mm}$ ): $\mathrm{NaCl} 479.12, \mathrm{KCl} 12.74$, $\mathrm{CaCl}_{2} 3.00$, and $\mathrm{HEPES} 5, \mathrm{pH} 7.45$, and then fixed with $4 \%$ ethyldimethyl-carbodiimide in low calcium physiological saline for $0.5 \mathrm{hr}$. To wash out the fixative, all preparations were then rinsed at least five times over at least $2 \mathrm{hr}$ in a solution of PBS with $0.3 \%$ Triton X-100 (PBST), $\mathrm{pH}$ 7.4. The preparations were then incubated in primary antibody for $24-48 \mathrm{hr}$ at $4^{\circ} \mathrm{C}$ and again rinsed at least five times over at least $2 \mathrm{hr}$ in PBST. Preparations were then incubated for $12-24 \mathrm{hr}$ at $4^{\circ} \mathrm{C}$ in goat anti-rabbit fluorescein-conjugated immunoglobulin (Sigma) diluted 1:200. Preparations were then rinsed in PBS before in toto acquisition of both retrograde dye migration and immunocytochemichal staining. All immunolabels were diluted in $10 \%$ normal goat serum PBST.

Confocal microscopy. All preparations were viewed directly in the dish with a Leica TCS 4D laser scanning confocal microscope equipped with a krypton/argon mixed gas laser. For the embryos, 20-30 optical sections, of thickness $1-1.5 \mu \mathrm{m}$, were recorded with a $50 \times$ water immersion objective. For the adults, 40-60 sections, of thickness 1-3 $\mu \mathrm{m}$, were recorded with a $10 \times$ or $20 \times$ air objective. Images presented were obtained using the maximal projection program provided by Scanware.

Quantitative analysis. Stained somata in each ganglion were counted on each of the sequential optical sections. All results were expressed as mean \pm SEM. Statistical comparisons among three groups (embryos, juveniles, and adults) were assessed by ANOVA on ranks followed by Dunn's test. Statistical comparisons between two groups (embryo vs adult; embryo vs juvenile) were assessed using the Mann-Whitney rank sum test. It must be noted that the photomicrographs illustrating the results do not necessarily match the mean number of stained somata reported.

\section{RESULTS}

The stomatogastric nervous system of the adult lobster H. gammarus consists of four interconnected ganglia: the STG, which contains two distinct motor networks and more rostral ganglia consisting of the esophageal ganglion (OG), and the pair of commissural ganglia (CoGs), which contain the somata of central projection neurons. The rostral ganglia are linked to the STG via a single nerve, the stomatogastric nerve (stn) and the two inferior (ion) and superior (son) nerves and the esophageal (on) nerve. These ganglia are also connected to the brain via the inferior ventricular nerve (ivn). The STG neurons reach their muscle targets via a common nerve, the dorsal ventricular nerve (dvn). This latter splits into two lateral ventricular nerves (lvns) that project to the muscles (Fig. 1A1).

The embryonic STNS can be visualized very early in development (Casasnovas and Meyrand, 1995) and can be dissected out as early as $65 \%$ of development (Fig. 1B2). Embryonic and adult STNSs possess a similar organization (Fig. 1, compare $A 1$ and $B 1)$. For example, the STG is linked to the more rostral ganglia by a single afferent nerve, the stn, which in the adult contains all the axons of central projection neurons. This anatomical feature let us use retrograde dye migration to trace these neurons during the course of development. However, embryonic and adult STNSs differ in size. For example, the whole embryonic STNS (Fig. 1B2) has a size similar to that of the whole adult STG (Fig. 1A2).

\section{Quantification and localization of embryonic and adult projection neurons to the STG}

In the adult, the activity of STG networks is controlled by central modulatory inputs arising from the rostral ganglia (OG, CoGs) through the stn (Harris-Warrick et al., 1992), and by sensory neurons located in the lvn that project to the STG via the dvn (Katz and Harris-Warrick, 1989). In H. gammarus many of these projection neurons have been identified, and the central input neurons have been studied extensively (Meyrand et al., 1991, 1994, 2000; Nagy et al., 1994; Combes et al., 1999). Many of the projection neurons are well characterized in terms of electrical activity, neurotransmitter phenotype, soma location (CoG, OG, ivn, and lvn), morphology, and projection pattern (Table 1). In the embryo, recent immunocytochemical detection of neuromodulators suggests that projection inputs to the STG appear gradually throughout development, although some are present very early in the development (Cournil et al., 1995; Fenelon et al., 1998a, 1999; Kilman et al., 1999). Furthermore, electrophysiological studies indicate that some of these projection neurons are present and functional early in development (Le Feuvre et al., 

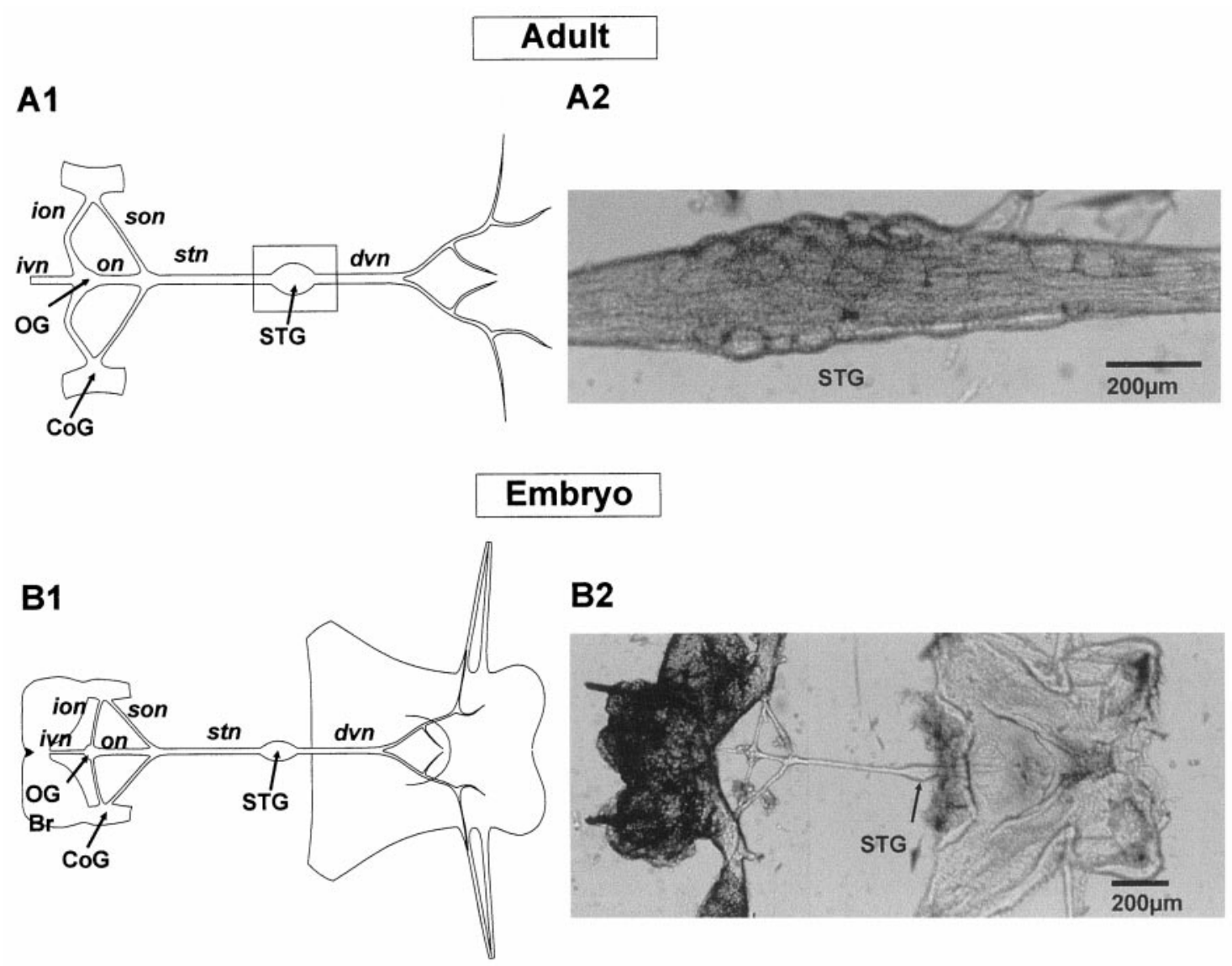

Figure 1. Similar organization of the adult $(A)$ and embryonic $(B)$ STNS of the lobster, $H$. gammarus. A1, Schematic representation of the dissected adult STNS in vitro. A2, Photomicrograph of the adult stomatogastric ganglion $(S T G)$. B1, Schematic representation of the embryonic nerve-muscle preparation. $B 2$, Photomicrograph of the dissected nerve-muscle embryonic preparation at $90 \%$ of development. In all figures, rectangles in the schematic drawings (here, $A 1)$ indicate the area covered by the photomicrographs. $\mathrm{Br}$, Brain; $\mathrm{CoG}$, commissural ganglion; $d v n$, dorsal ventricular nerve; ion, inferior esophageal nerve; $i v n$, inferior ventricular nerve; $O G$, esophageal ganglion; on, esophageal nerve; son, superior esophageal nerve; stn, stomatogastric nerve.

\begin{tabular}{|c|c|c|c|c|}
\hline $\begin{array}{l}\text { Name of projecting neuron } \\
\text { (number of cells) }\end{array}$ & Soma location & Axon location & Projection & References \\
\hline PS (2) & ivn & ivn, ion, on, son, stn & STG, CoG & Cazalets et al., 1990; Meyrand et al., 1994 \\
\hline GN1/2 (2) & OG/on & on, son, stn & STG & Cournil et al., 1990 \\
\hline GN3/4 (2) & OG/ion & ion, son, stn & STG & Cournil et al., 1990 \\
\hline CD1 (1) & OG & ion, on, stn & STG & Present study \\
\hline GN5/6 (4) & $\mathrm{CoG}$ & ion, on, stn & STG & Cournil et al., 1990 \\
\hline $\mathrm{CG}(1)$ & $\mathrm{CoG}$ & son, stn & STG & Simmers et Moulins, 1988 \\
\hline GI (1) & $\mathrm{CoG}$ & son, stn & STG & Combes et al., 1999 \\
\hline $\mathrm{CP}(1)$ & $\mathrm{CoG}$ & son, stn & STG & Nagy et al., 1994 \\
\hline GPR $(6-12)$ & $\operatorname{lvn}$ & dvn, stn & STG & Present study \\
\hline
\end{tabular}

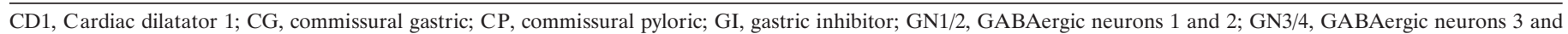

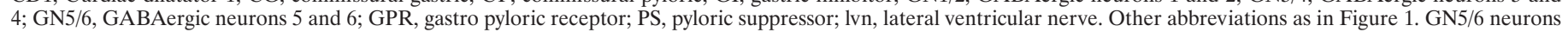
were identified as P and a cells (Nagy et al., 1994) using electrophysiological techniques.

1999). Therefore, to determine which of the known adult projection neurons are already present in the embryo, we performed retrograde dye migration from stn toward the anterior ganglia, and from the dvn toward the muscles, in the embryo, juveniles, and adult animals.

Neurons in the CoGs that project to the STG

The adult STG networks receive modulatory input from neurons located in the CoGs. To identify $\mathrm{CoG}$ neurons projecting to the
STG via the stn, we performed retrograde dye migration from the stn toward the CoG (Fig. 2A1). The stained somata were counted on sequential optical sections of CoGs to distinguish cells with similar location but in different planes, whereas the pictures presented in all Figures are maximal projections from several optical sections. In the adult, the distance between the stn and the CoGs is too long to perform dye migration, and most experiments were therefore performed on juveniles. Dye retrograde migration from the stn toward the CoGs via the ion and son (Fig. 2A1) 
A1
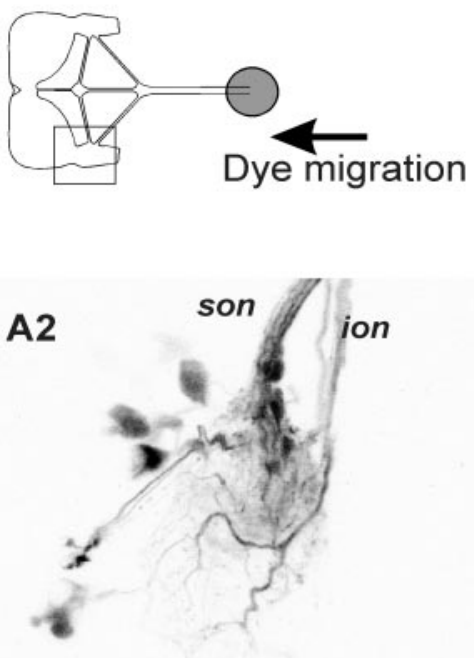

$20 \mu \mathrm{m}$

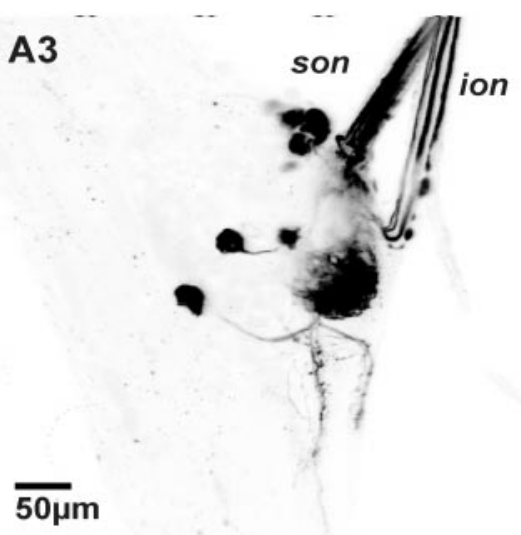

A4

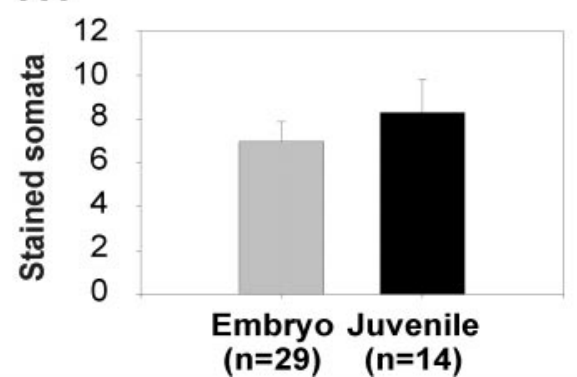

B1
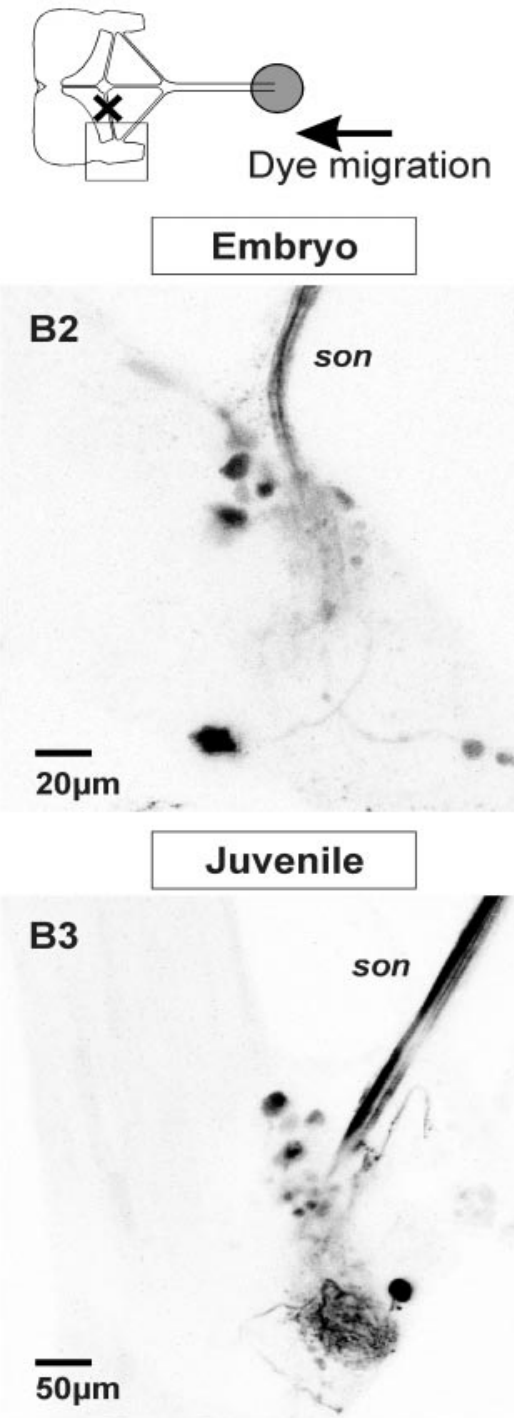

B4

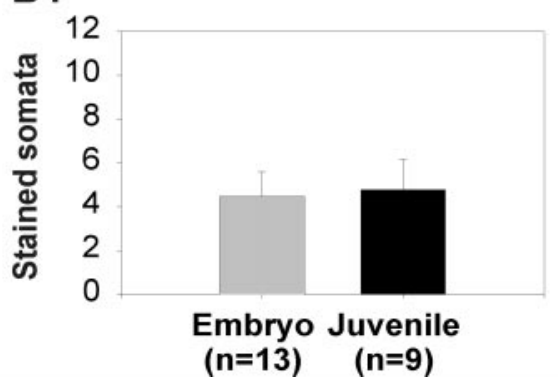

C1

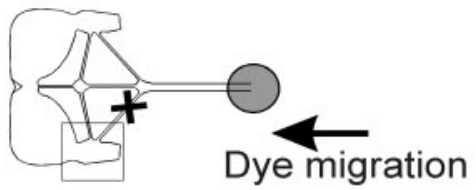

C2
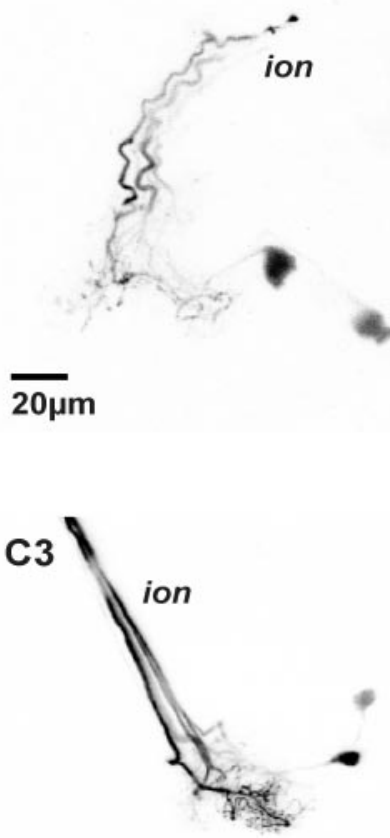

$50 \mu \mathrm{m}$

C4

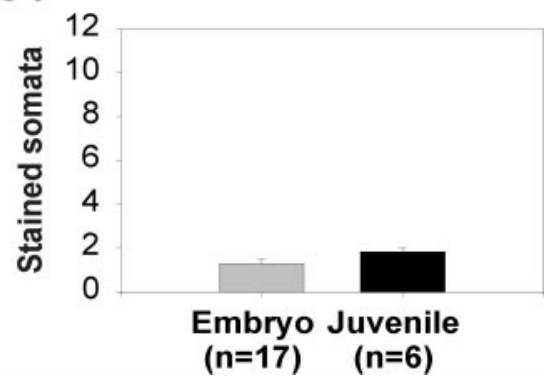

Figure 2. CoG neurons projecting to the STG and their projection pattern. Retrograde dye migration performed from the stn toward the anterior ganglia $(A 1)$ stained $\sim 10$ neuronal somata in the embryonic $(A 2)$ and juvenile $(A 3)$ CoG. The same migration performed after cutting the ion (B1) stained approximately six to eight somata in both embryo (B2) and juvenile (B3), whereas when the son was cut, dye migration stained typically two somata within each CoG, in both the embryo $(C 2)$ and the juvenile $(C 3)$. Quantitative analysis of these data showed no statistically significant difference (Mann-Whitney rank sum test) between the number of stained somata in embryonic and juvenile CoGs, whereas anterior nerve was maintained intact for migration ( $A 4$, both son and ion; $B 4$, son only; $C 4$, ion only).

labeled up to 19 neurons in the embryo (range, 0-19; mean, $6.97 \pm 0.91 ; n=29$ ) (Fig. $2 A 2, A 4)$ and up to 16 in the juvenile CoGs (range, $0-16$; mean, $8.28 \pm 1.51 ; n=14$ ) (Fig. $2 A 3, A 4$ ). There was no significant difference between the number of labeled somata in embryo and juvenile (Fig. 2A4).
In the adult, most CoG neurons projecting to the stn send their axons to the stn via the son, and only two of them extend axons into the ion (Nagy et al., 1994). We tested whether embryonic CoG neurons have a projection pathway similar to those in the adult by performing dye migration from the stn toward the ante- 
A
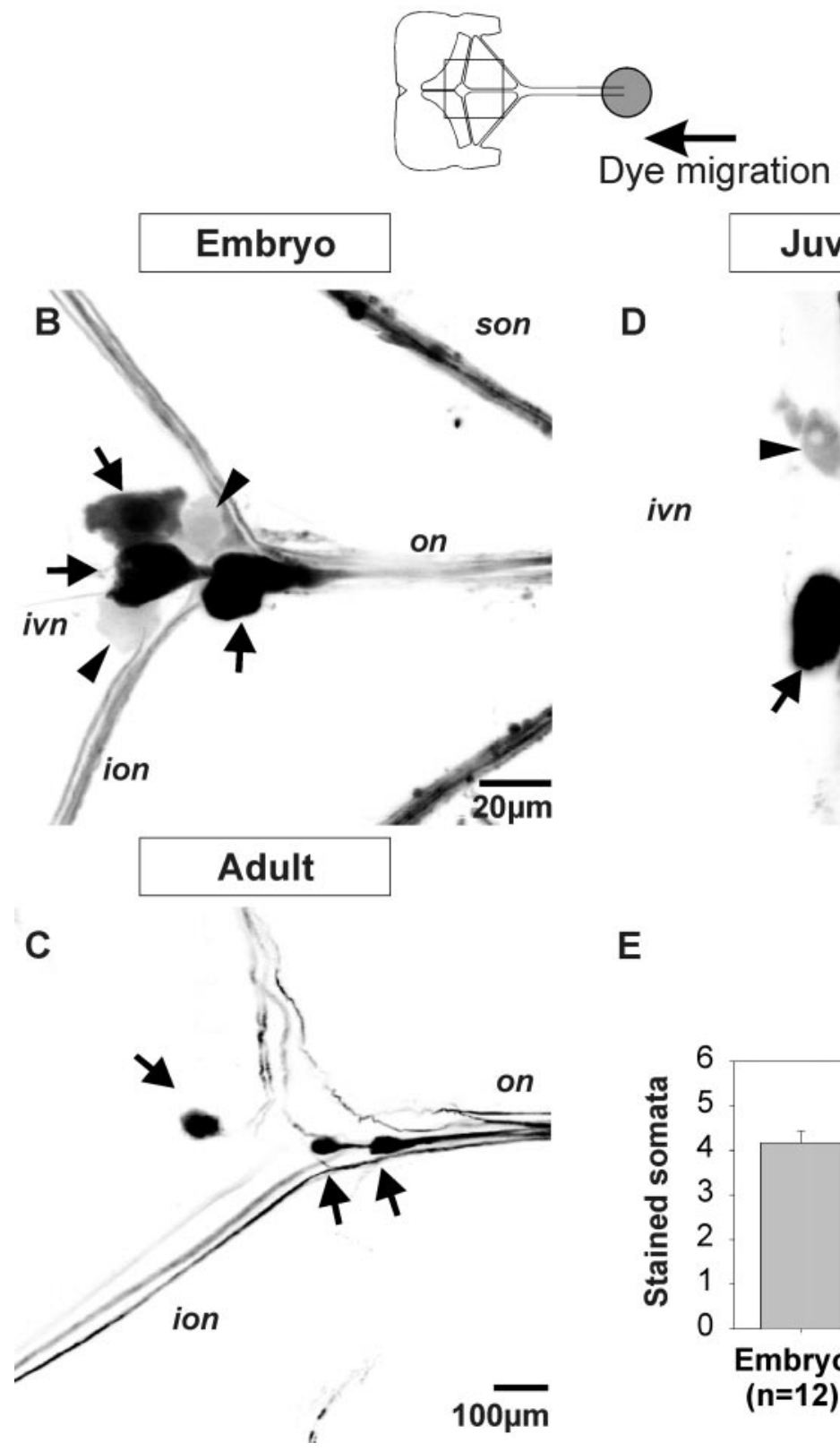

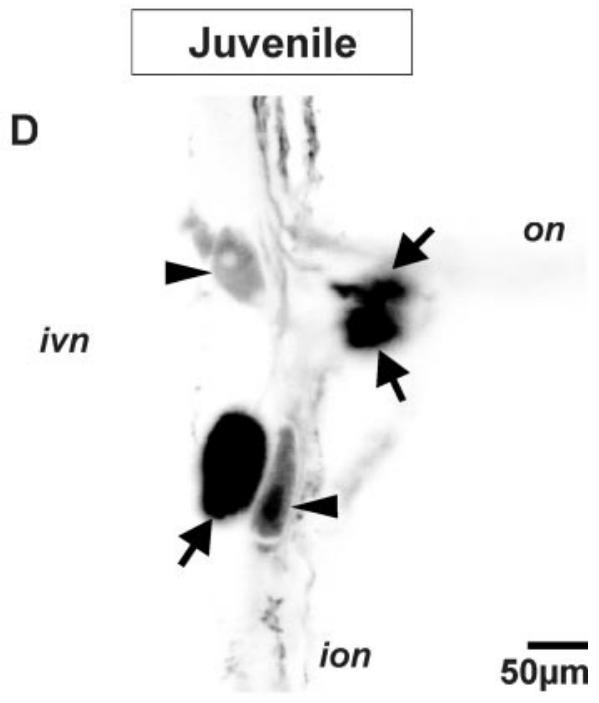

E

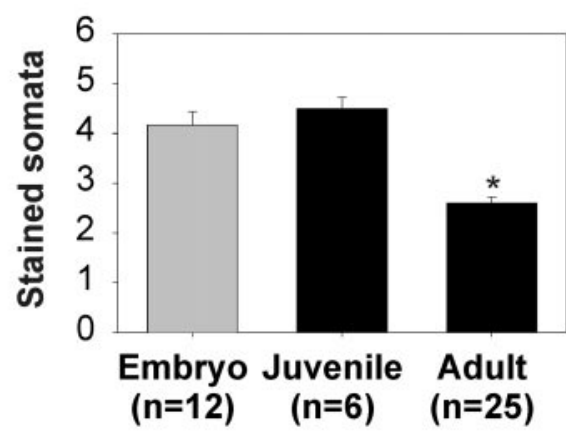

Figure 3. OG neurons projecting to STG in embryo, juvenile, and adult: quantitative analysis. Retrograde dye migration performed from the stn $(A)$ toward the OG typically stained five neuronal somata in the embryonic OG $(B)$. Three of these neurons were strongly stained by dye migration (arrows), whereas the other two were generally less intensely stained (arrowheads). In the adult $(C)$, only three strongly stained somata were found in the OG (arrows), but in juveniles ( $D$ ), five somata in the OG were stained, two of them being stained less intensely (arrowheads). The mean number of stained somata $(E)$ in the adult OG was statistically lower than in embryonic and juvenile OG (ANOVA on ranks followed by Dunn's test; * $p<0.05$ vs embryo and juvenile). rior ganglia after cutting either the ions (Fig. 2B1) or the sons (Fig. 2C1). We found that in the embryo, up to 13 (range, 0-13; mean, $4.46 \pm 1.12 ; n=13) \mathrm{CoG}$ neurons project to the stn via the son (Fig. 2B2) and only 2 (range, $0-2$; mean, $1.29 \pm 0.18 ; n=17$ ) via the ion/on pathway (Fig. 2C2). Similarly, in juveniles, we found that up to 14 neurons project from the CoG to the stn via the son (range, $0-14$; mean, $4.78 \pm 1.4 ; n=9)($ Fig. $2 B 3, B 4)$ and only 2 via the ion/on (range, $0-2$; mean, $1.83 \pm 0.16 ; n=6$ ) (Fig. $2 C 3, C 4)$. Counts of the mean number of stained somata revealed no statistical differences between embryos and juveniles in the mean number of cells projecting from the $\mathrm{CoG}$ to the stn, whatever their pattern projection (Fig. $2 A 4, B 4, C 4$ ). In the adult, the two somata projecting to the stn via the ion/on pathway were identified as a GN5/6 pair of projection neurons (Cournil et al., 1990), one of which is also called the P cell (Nagy et al., 1994). Therefore, the two CoGs somata stained through the stn/on/ion pathway in the embryonic preparation appear to correspond to the identified neurons GN5/6. Although the cells projecting through the son could not be morphologically identified in either the embryo or the juvenile, our data indicate that an equivalent population of neurons project from the CoGs to the stn through the son or ion in the embryo and juvenile.

\section{Neurons in the OG that project to the STG}

In addition to CoG input neurons, adult STG networks also receive modulatory inputs from neurons located in the vicinity of the OG. To determine whether these neurons are also present in the embryo, we performed retrograde dye migration from the stn toward the OG (Fig. $3 A$ ). In the embryo, such migration typically stained five neuronal somata (range, 3-5; mean, $4.17 \pm 0.27 ; n=$ 12), two of them being weakly stained (Fig. $3 B$, arrowheads). By contrast, in the adult, only three cells (range, 1-3; mean, $2.60 \pm$ 
A1

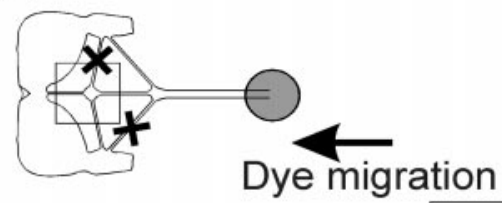

B1

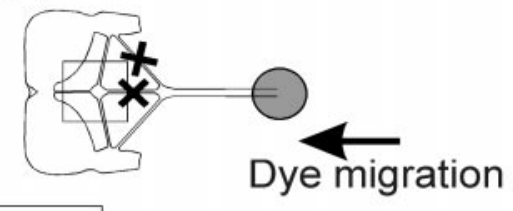

\section{Embryo}

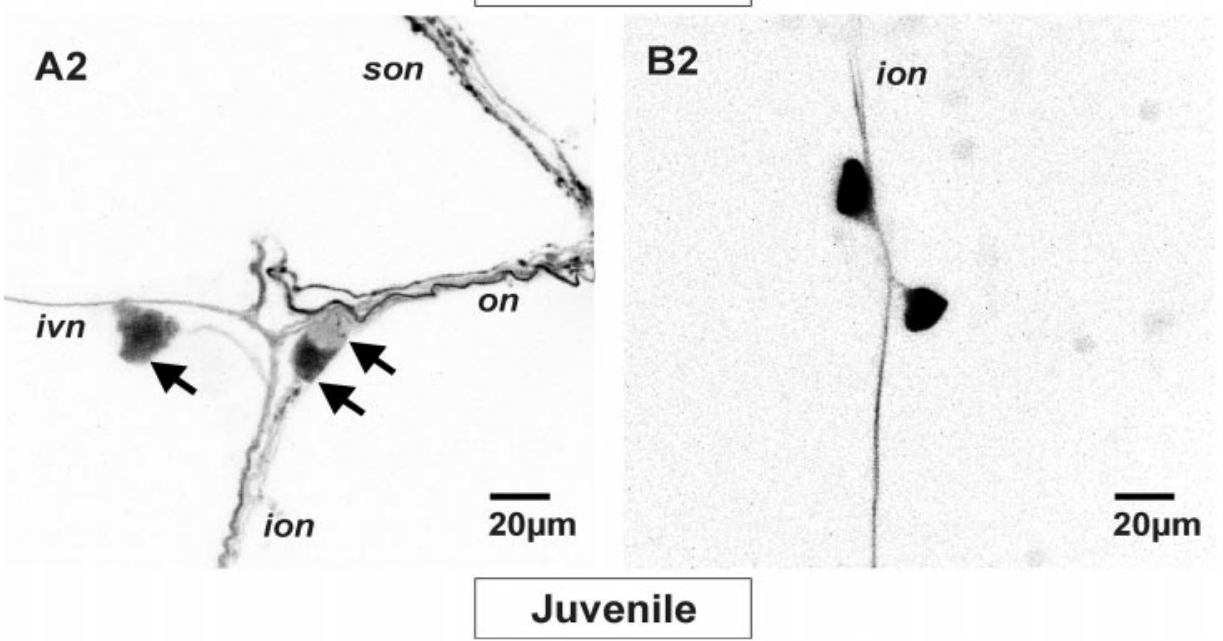

Figure 4. OG neurons projecting to STG in embryo and juvenile: projection pattern. Retrograde dye migration performed from the $s t n$ after section of one ion and its contralateral son (A1) stained three neuronal somata in the embryonic $(A 2)$ and juvenile $(A 3)$ OG. Two of them had fusiform somata and projected in the on and both sons. The outline of one such neuron in the embryo is illustrated in $A 4(i)$ (taken from a preparation different from that shown in A2). The last neuron had a globular soma and projected in both ions and on. The outline of this cell in the embryo is illustrated in $A 4$ (ii) (taken from a preparation different from that shown in $A 2$ ). Retrograde dye migration performed from the $s t n$ after section of the on and one son (B1) stained two neuronal somata in the embryonic (B2) and juvenile (B3) OG. The projection pattern of these cells in the embryo is illustrated in $B 4$ (drawing from $B 2$ ).
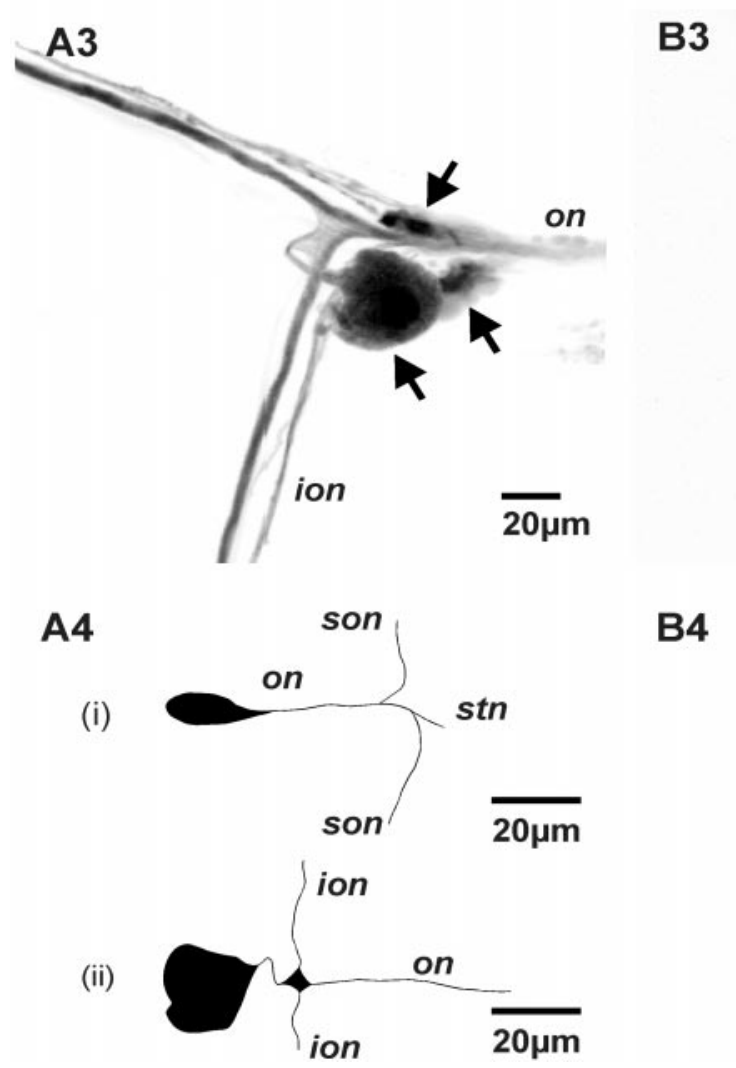

\section{B4}

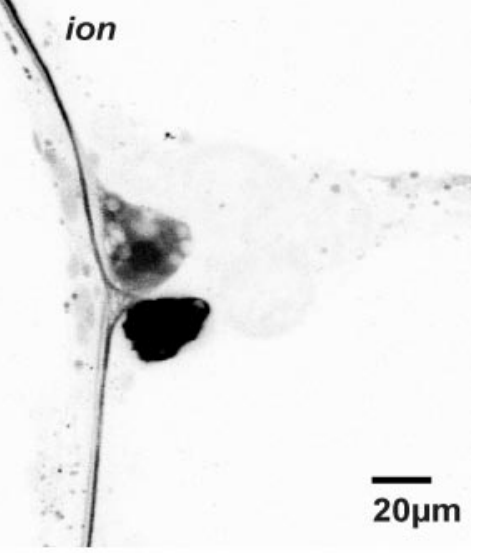

$0.11 ; n=25$ ) were labeled in the OG (Fig. 3C). In the adult, besides the three neurons already stained, two additional projection neurons (GN3/4) with somata located in the vicinity of the OG send axons to the STG symmetrically via both ions and sons, and then stn (Cournil et al., 1990). However, because of the size of the preparation, in the adult the somata of these neurons have never been stained using retrograde dye migration from the stn. Therefore we used juveniles to check whether these additional cells could be stained in small-sized animals. Dye migration from the stn toward anterior ganglia stained five neuronal somata in the juvenile OG (range, $4-5$; mean, $4.50 \pm 0.22 ; n=6$ ) (Fig. 3D). Comparison of the mean number of stained somata in the embryo, juvenile, and adult (Fig. $3 E$ ) showed a significant difference (Dunn's test, $p<0.05$ ) between the adult and the juvenile and between the adult and the embryo, whereas there was no statistical difference between the embryo and the juvenile. 
A
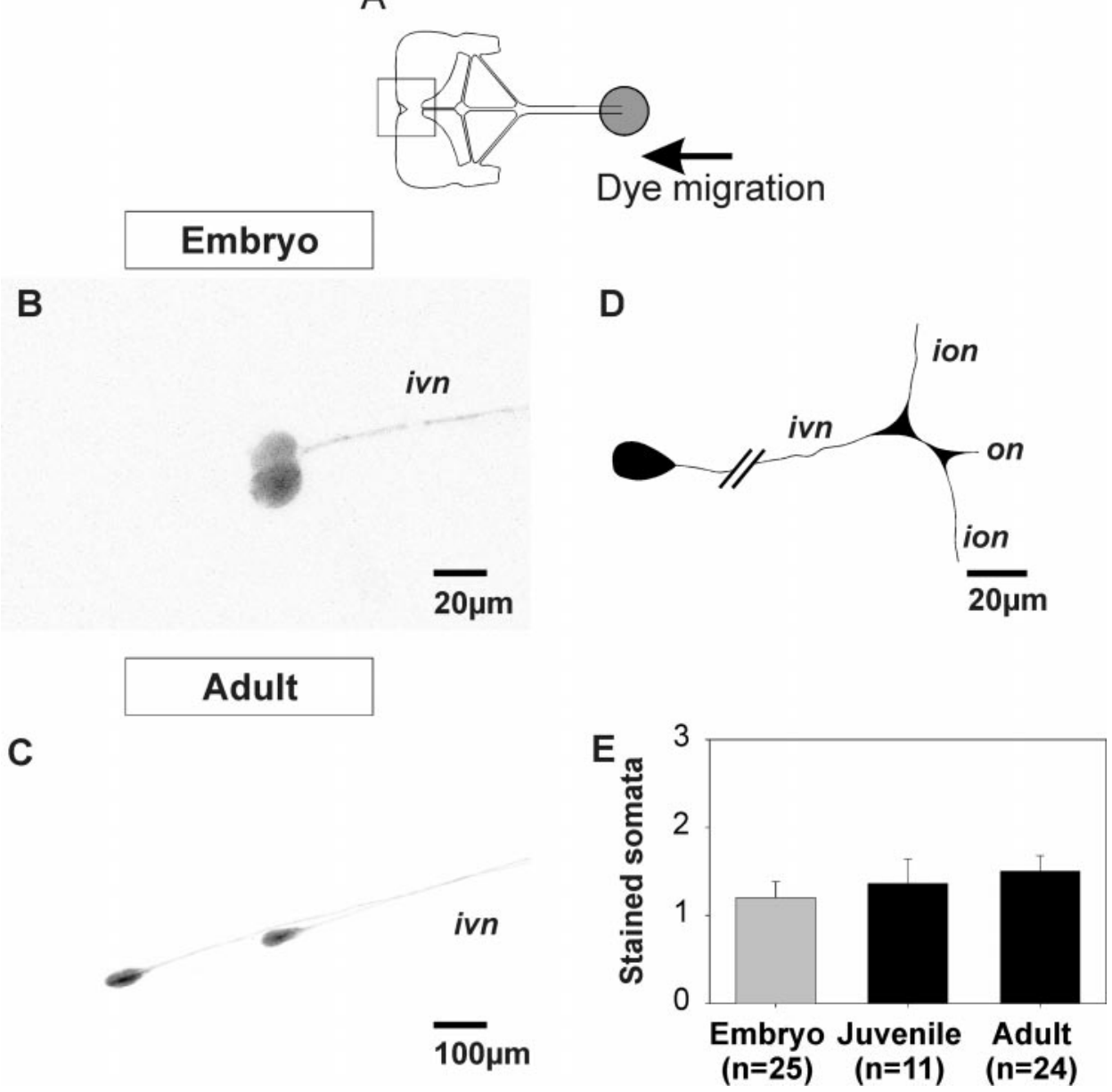

Figure 5. ivn neurons projecting to the STG. Retrograde dye migration performed from the stn toward the OG typically stained two neuronal somata in the brain at the emergence point of the ivn (B, Embryo), or in the ivn $(C, A d u l t)$. The projection pattern of these neurons is drawn in $D$ from a different embryonic preparation than $B$. The mean number of stained somata $(E)$ in the embryo, juvenile, and adult was not significantly different (ANOVA on ranks).

We then determined the projection pattern of the OG neurons in the embryo and the juvenile. OG neurons could project to the stn either directly through the on or indirectly through the ion and then son. Therefore, to exclusively stain the OG neurons projecting to the STG via the on, we performed dye migration from the stn toward the $\mathrm{OG}$ after section of two anterior nerves (son/son or ion/ion or son/contralateral ion) (Fig. 4A1). In these conditions, only three neuronal somata in the embryo (Fig. 4A2) $(n=11)$ as well as the juvenile (Fig. $4 A 3)(n=9)$ were reliably stained. The projection pattern of $\mathrm{OG}$ neurons was also examined in dye migration experiments from the stn with the on and one son cut. Although the on section should prevent the staining of the three former somata, the son section will test the symmetrical projection pattern of the two remaining projection neurons (see above). In such a condition (Fig. $4 B 1)(n=4)$, two somata were reliably found in embryo (Fig. 4B2) and juvenile (Fig. 4B3). Together, these data indicate that in the embryo, juvenile, and adult (Cournil et al., 1990), five OG neurons project to the STG.

These OG neuronal somata display similar locations and projection patterns in the embryo, juvenile, and adult. Two of them were unipolar fusiform cells, located near the on entrance, that sent a single axon in the on and also projected in the two sons (Fig. 4A4i). A globular soma located near the ivn entrance had a single axon that split in the OG into three main processes projecting in the on and the two ions (Fig. 4A4ii). In the adult, the two fusiform cells projecting in the on and sons have been identified as GN1/2 modulatory neurons (Cournil et al., 1990), and the globulous one, projecting in both ions and on, has been identified as the species-equivalent version of the CD1 motoneuron (Nagy, 1981). The two remaining OG projection neurons had a short neurite that emerged from the cell body and divided into two processes that projected into both ions (Fig. 4B4) and then into the sons (data not shown). These neurons possess similar soma locations and pattern projections as the projection neurons GN3/4 (Cournil et al., 1990). These data demonstrate that the adult $\mathrm{OG}$ projection neurons seem to be present in the embryo and express the same projection pattern.

Retrograde migration from the stn toward the OG (Fig. 5A) also typically labeled two neuronal somata $(1.20 \pm 0.18, n=25$ for the embryo; $1.36 \pm 0.28, n=11$ for the juveniles; $1.50 \pm 0.18$, $n=24$ for the adult; range, $0-2$ in all cases) in the brain at the emergence point of the ivn in the embryos (Fig. $5 B$ ), or in the ivn in the adult (Fig. $5 C$ ). In the juveniles, these neurons were located either in the brain (see Fig. 8A2) or in the ivn (see Fig. 8A4). Statistical analysis showed no significant difference in the number of stained somata at this location in the adult, juvenile, and embryo (Fig. $5 E$ ). In both the adult (Fig. $5 C$ ) and embryo (Fig. $5 B)$, these cells were monopolar cells that sent a single axon in the ivn toward the OG. In the embryonic OG, this axon later split into three neuronal processes projecting into the on and ions (Figs. $4 A 2,5 D$ ). In the adult, these cells were identified as pyloric suppressor (PS) neurons (Cazalets et al., 1990). Comparison of soma location, soma morphology, and projection pattern suggests that these embryonic cells correspond to the PS modulatory neurons.

These data support the hypothesis that the entire identified 

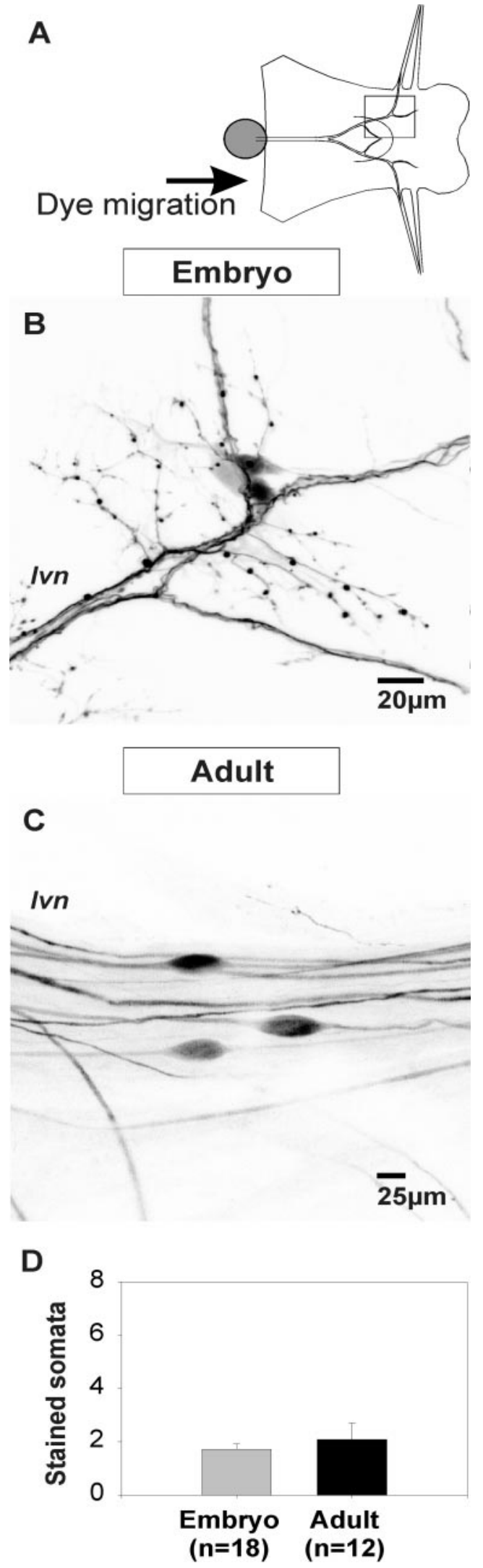

Figure 6. Peripheral sensory neurons that project to the STG. Retrograde migration performed from the $\operatorname{dvn}(A)$ in the embryo $(B)$ or the $l v n$ in the adult $(C)$ typically stained three to four neuronal somata at the location indicated in $A$ (rectangle). The mean number of stained somata $(D)$ in the embryo and adult displayed no statistically significant difference (Mann-Whitney rank sum test). population of projection neurons located in the anterior ganglia and projecting to the adult STG is already established in the embryo at $65 \%$ development.

\section{Neurons in the periphery that project to the STG}

In the adult, besides the central modulatory input neurons, the STG also receives input from peripheral sensory neurons. In the $\mathrm{crab}$, these sensory neurons, the gastropyloric receptors (GPRs) (Katz et al., 1989), have their cell body located in the peripheral nerves innervating the gastropyloric muscles and send their axon to the STG via the lvn and dvn and to more anterior ganglia via the stn. To assess whether these neurons are also present in Homarus and express similar projection patterns in the embryo and adult, we performed retrograde dye migration from the dvn (Fig. 6A, Embryo) or the lvn (Adult) toward the gastropyloric region.

In the embryo, retrograde migration performed from the dvn toward the pyloric muscles typically labeled up to three neuronal somata at the entrance of the pyloric part of the stomodeum $(1.72 \pm 0.21 ; n=18$; range, $0-3)$ (Fig. $6 B, D)$. In the adult, the same migration performed from the lvn toward the muscles typically labeled up to six neuronal somata in the lvn at the gastropyloric valve level $(2.08 \pm 0.60 ; n=12$; range, $0-6)$ (Fig. $6 C, D)$. Here, as for the other input neurons to the STG (see above), there was no statistical difference between the number of labeled peripheral somata in the embryo and the adult (Fig. 6D). Furthermore, in the embryo, when we performed dye migration from the stn toward STG, the same cluster of peripheral cells was stained (data not shown), indicating that they also project to anterior ganglia via the stn (as reported in Cancer borealis) (Katz and Harris-Warrick, 1989; Katz et al., 1989). Most of these cells had bipolar soma, in both the embryo and the adult. In a previous immunocytochemical study, there were a maximum of six FLRFamide-positive neurons, identified as GPR neurons, found in the embryonic, larval, and adult lvn posterior to the gastric mill muscles of $H$. americanus and gammarus (Kilman et al., 1999). Thus, we conclude that the stained neurons in the present paper are the embryonic and adult GPR neurons, already characterized using either immunocytochemichal (Beltz et al., 1984; Katz et al., 1989; Turrigiano and Selverston, 1991; Kilman et al., 1999; Skiebe, 1999) or electrophysiological (Katz and Harris-Warrick, 1989; Birmingham et al., 1999) techniques. Therefore, our data indicate that GPR cells are already present at developmental stage $65 \%$ and that they express the same pattern of projection as in the adult.

In summary, these results show that the known population of central and peripheral projection neurons to the STG is quantitatively established as early as $65 \%$ of development. This contrasts with previous demonstrations that neuromodulatory phenotypes are acquired gradually throughout development (Fenelon et al., 1998a, 1999; Kilman et al., 1999). Therefore, ontogenetic plasticity seems to depend on changes in transmitter phenotype expression within the same early established population of projection neurons rather than on a progressive acquisition of new projection fibers or neurons. To understand the individual process of acquisition of neurotransmitter phenotype within a population of projection neurons, we performed immunocytochemical detection of neuromodulatory substances after labeling neurons by dye migration. 

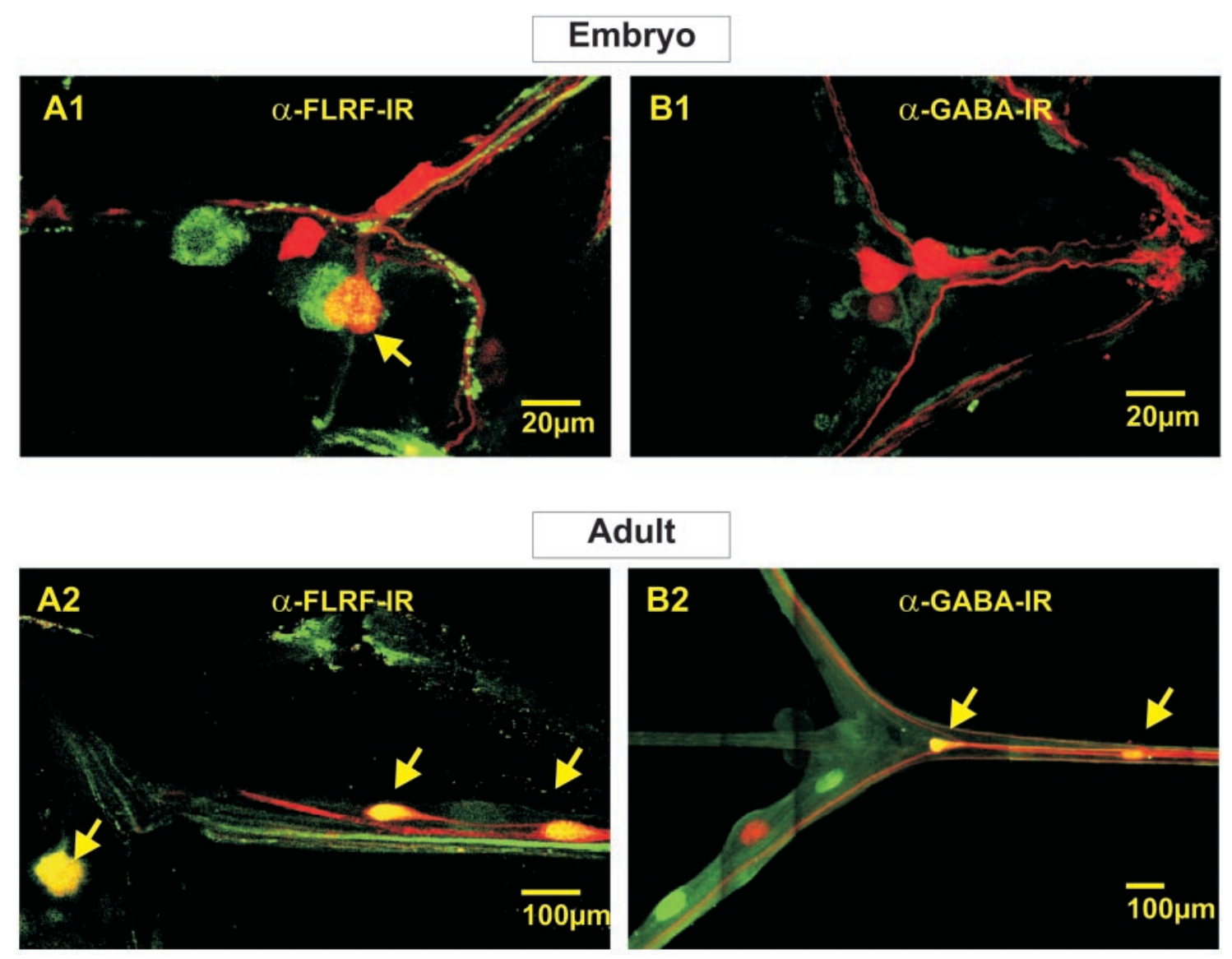

Figure 7. FLRFamide- and GABA-like immunoreactivity in embryonic and adult identified OG neurons. Retrograde dye migration (red staining) from the stn toward the OG was coupled with subsequent immunocytochemical detection (green staining) of either FLRFamide-like peptides ( $A$, anti-FLRFamide immunoreactivity: $\alpha$-FLRF-IR) or GABA ( $B$, anti-GABA immunoreactivity: $\alpha$-GABA-IR), whereas in the embryo $(A 1)$, only one (yellow arrow) of the three neurons projecting into the stn exhibited $\alpha$-FLRF-IR; in the adult (A2), all three neurons projecting into the stn displayed $\alpha$-FLRF-IR (yellow arrows). The two additional FLRFamide-immunoreactive cells in the embryonic OG (A1) were also present in the adult OG (A2), but outside the frame of the picture. Furthermore, in the embryo (B1), none of the cells projecting to the STG showed $\alpha$-GABA-IR, whereas in the adult (B2), the two fusiform neurons projecting into the stn were GABAergic (yellow arrows). Note that two additional GABAergic cells were present in the adult $\mathrm{OG}(B 2)$, whereas no GABA staining was observed in the embryonic OG (B1).

\section{Comparison of neurotransmitter phenotype of embryonic and adult projection neurons to the STG}

We focused our attention on neuromodulatory substances known to be expressed by some adult projection neurons that can be reliably identified in the embryo. We have shown previously that among the earliest detectable modulatory substances in the embryo [FLRFamide, proctolin, and Red Pigment concentrating hormone (RPCH)], only FLRFamide is commonly expressed in the adult by identified neurons such as GPR cells (Kilman et al., 1999), GN1/2 neurons (Meyrand et al., 2000), and PS neurons (Fenelon et al., 1998a). In contrast, although RPCH and proctolin immunoreactivity have been detected in the STG, so far the detection of these substances has not been coupled to electrophysiological identification of known projection neurons. Therefore we focused our attention on the three groups of projection neurons (GPR, GN1/2, PS) that have been shown to express FLRFamide immunoreactivity. Moreover, these neurons also express cotransmitters in the adult, such as serotonin for the GPR cells (Kilman et al., 1999) and GABA for GN1/2 neurons (Cournil et al., 1990; Meyrand et al., 2000), and in other species PS neuron homologs display histamine immunoreactivity (Mulloney and Hall, 1991). Previous indirect studies suggest that, for example, the GPR neurons gradually acquire their neurotransmitter
(FLRFamide, allatostatin, and serotonin) phenotype during development (Kilman et al., 1999). We therefore used retrograde dye migration with fluorescein immunocytochemical detection of FLRFamide, GABA, and histamine to assess whether these neurons express the same neuromodulators in the embryo and juvenile/adult.

In the embryo, retrograde labeling of OG neurons GN1/2 and CD1 followed by immunocytochemical detection of FLRFamide showed that only the CD1 motoneuron expresses FLRF immunoreactivity. Indeed, as illustrated in Figure 7A1, CD1 soma (indicated by the yellow arrow) clearly show large yellow areas resulting from the superposition of back fill staining (red) and FLRFamide immunoreactivity (green). In contrast, GN1/2 neurons appear homogeneously stained in red because of retrograde dye migration. This indicates that GN1/2 neurons do not express FLRFamide peptides, although absence of FLRFamide immunoreactivity may result from very low expression levels, below detection threshold. Moreover, two other FLRFamide immunoreactive cells are present in the OG (green somata, one of them close to CD1 soma). In the adult, both the CD1 motoneuron and the pair of GN1/2 modulatory neurons are FLRFamideimmunoreactive (Fig. 7A2, yellow arrows). As published previously (Fenelon et al., 1998a), three FLRF immunoreactive cells 


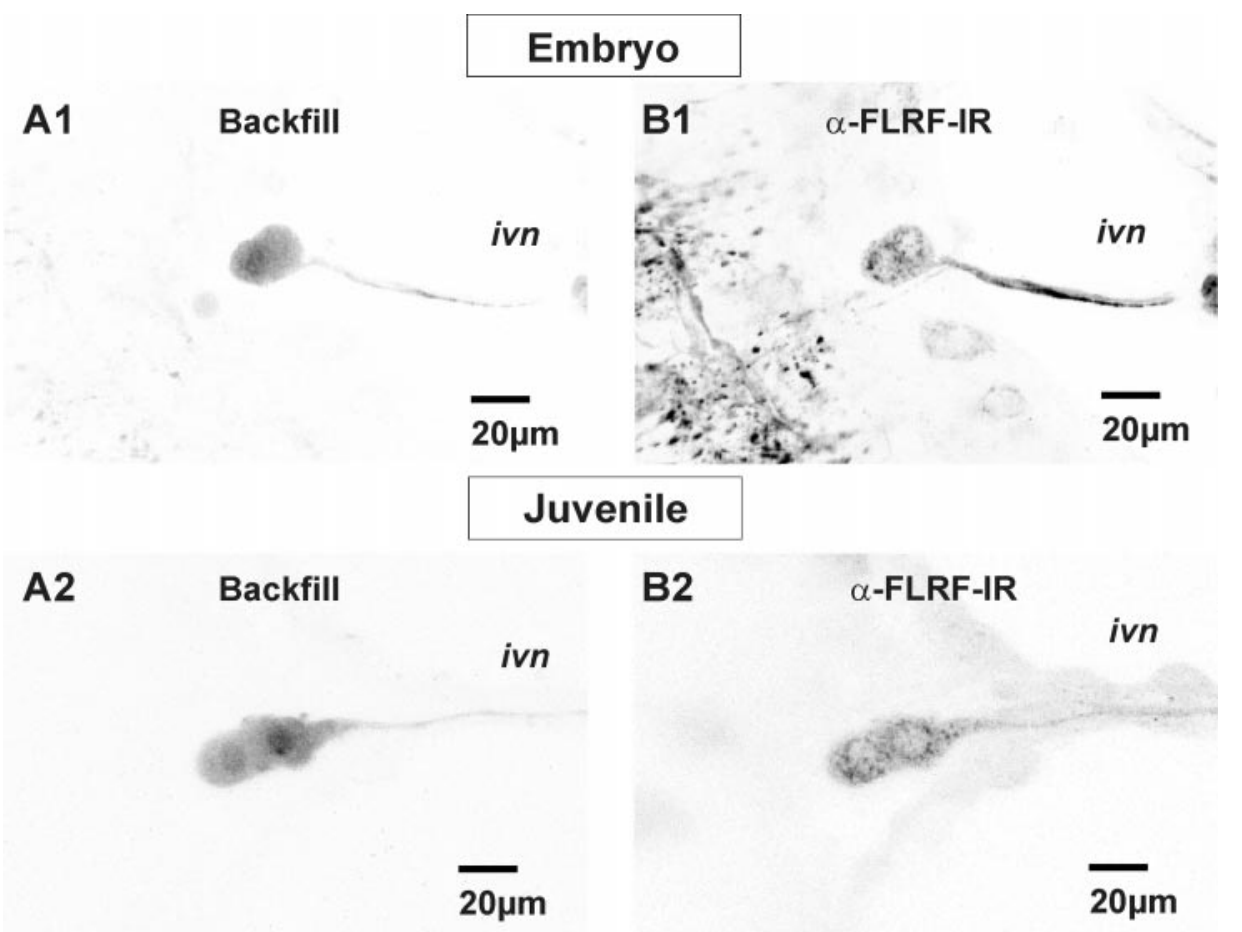

Figure 8. FLRFamide- and histamine-like immunoreactivity in embryonic and adult ivn neurons. Retrograde dye migration from the stn toward the ivn stains two somata in the brain at the emergence point of the ivn (A1, A3, Embryo; $A 2$, some juveniles), or in the ivn in some juveniles $(A 4)$. These neurons show FLRFamidelike immunoreactivity $(\alpha-F L R F-I R)$ in the embryo (B1) and the juvenile (B2). Similarly, these neurons also exhibit histamine-like immunoreactivity $(\alpha$-Histamine-IR) in the embryo (B3) and juvenile (B4). As a result of weakening of backfill staining intensity after carbodiimide fixation for histamine immunocytochemistry, $A 3$ and $A 4$ are confocal images acquired before immunocytochemical treatment shown in $B 3$ and $B 4$.

A3

\section{Backfill}

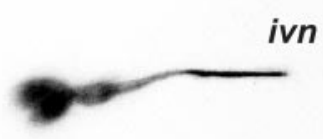

A4

Backfill

\section{Embryo}

\section{B3}

\section{$\alpha$-Histamine-IR}

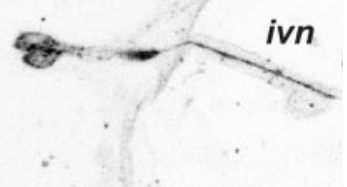

$20 \mu \mathrm{m}$

$20 \mu \mathrm{m}$

\section{Juvenile}

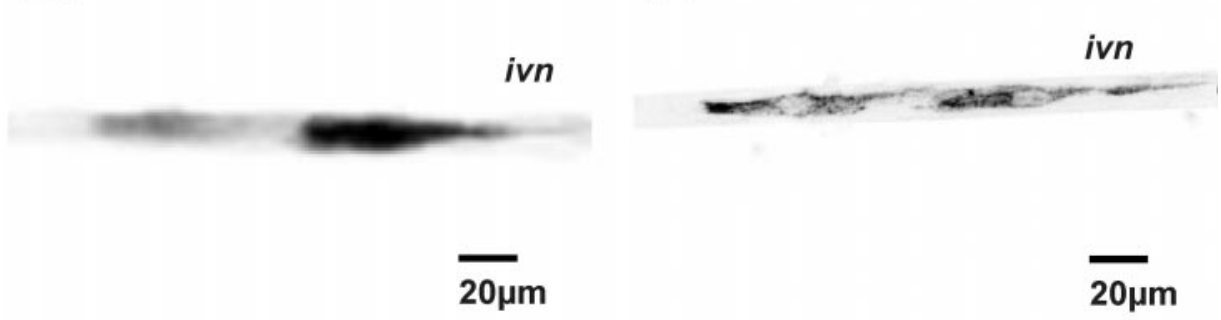

were present in the embryonic OG, whereas five were present in the adult OG (the two remaining FLRFamide-positive cells in the adult are outside the frame of the adult photomicrograph shown in Fig. 7A2). Moreover, no GABA staining was detected in the embryonic OG (Fig. 7B1), whereas the GN1/2 modulatory neurons are GABA immunopositive in the adult (Fig. 7B2, yellow arrows). Two additional GABA immunoreactive cells found in the adult ions (Fig. 7B2, green somata) were identified as GN3/4 neurons (Cournil et al., 1990). Moreover, in Figure 7B2, CD1 soma stained in red after back fill from the stn clearly do not display GABA immunoreactivity.

By contrast with the GN1/2 neurons, embryonic PS neurons stained by retrograde dye migration from the stn toward the ivn (Fig. 8A1, Embryo, A2, Juvenile) showed FLRFamide-like immunoreactivity as early as $65 \%$ development (Fig. $8 B 1$ ) as did juvenile (Fig. 8B2) and adult (data not shown) PS neurons. 

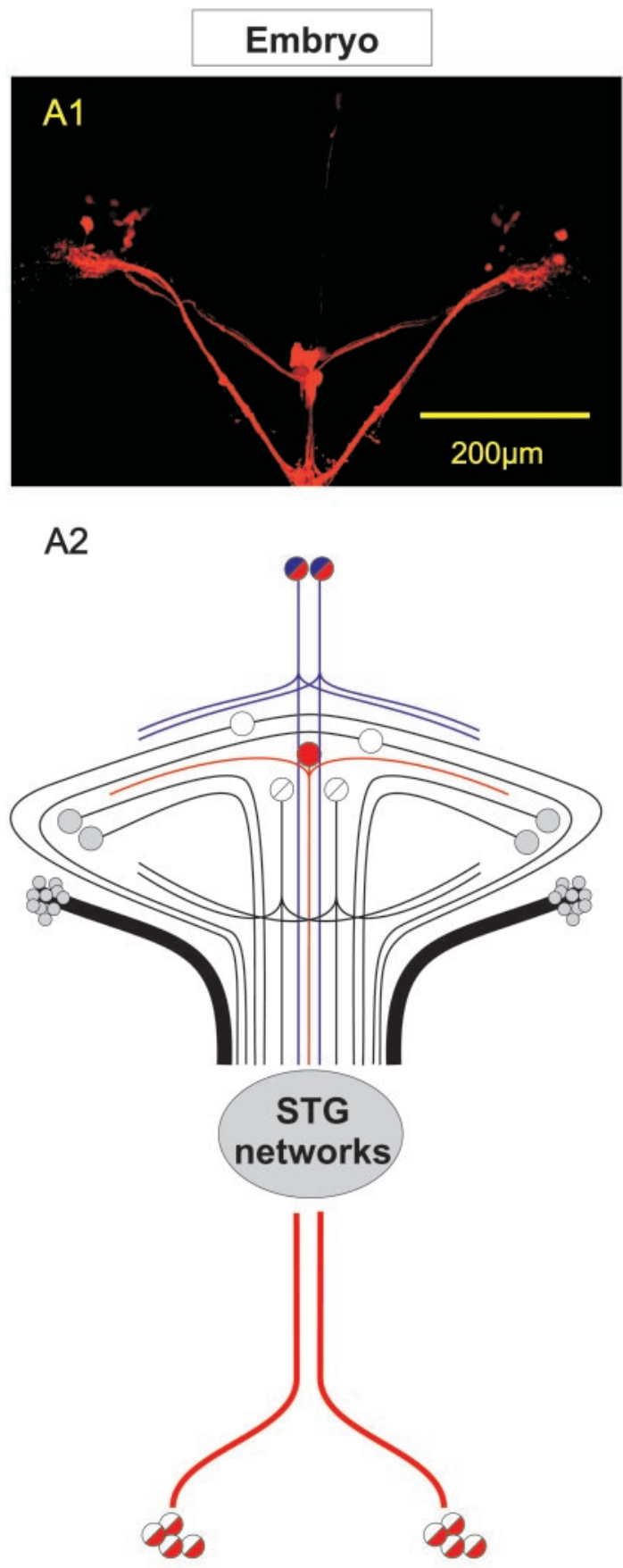

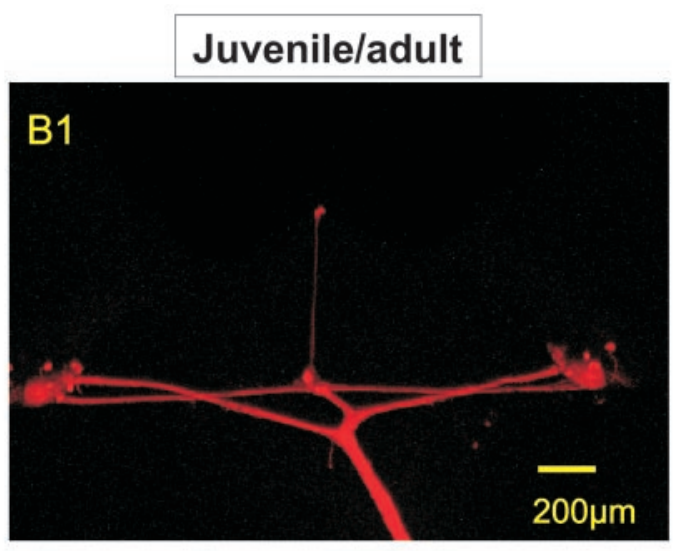

B2
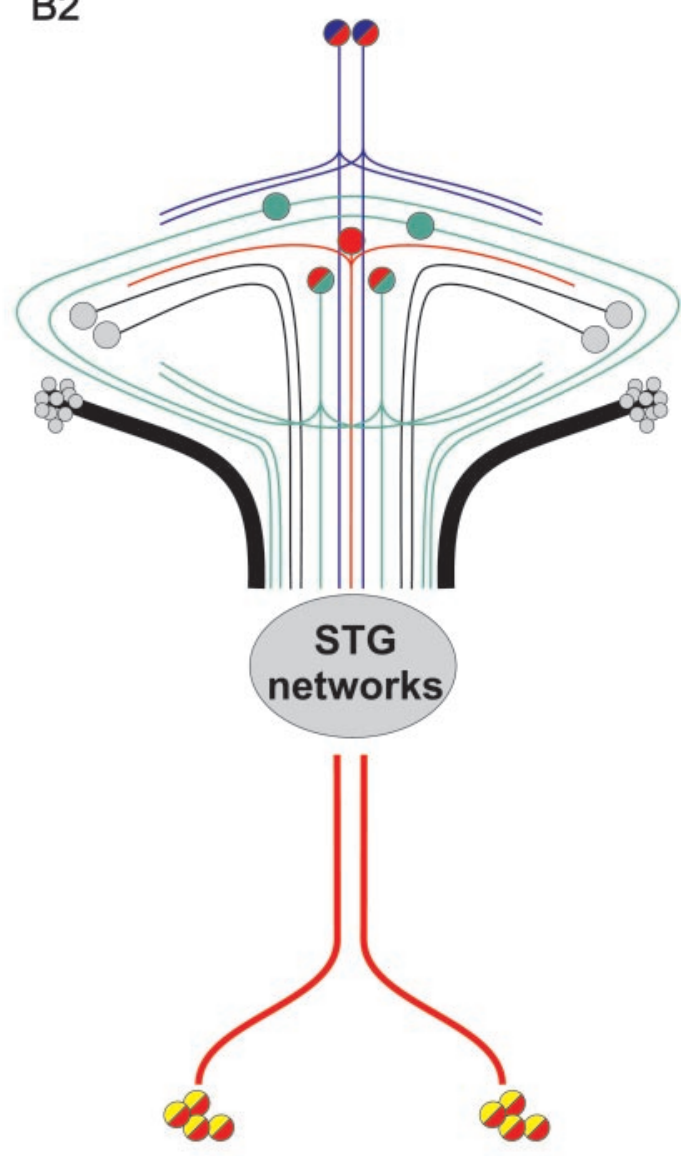

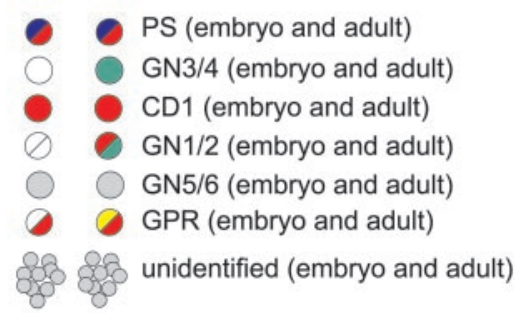

Figure 9. Delayed acquisition of neurotransmitter phenotype within an early established population of projection modulatory neurons. The cellular architecture of the modulatory input system is established early in development, with similar cell location and projections in the embryo $(A 1)$ and juvenile (B1). However, some of the embryonic neurons (A2) do not yet express their adult neurotransmitter phenotype (B2). $A 1$ and $B 1$ are reconstructions of the population of projection neurons to the STG, as revealed by dye migration techniques. $A 2$ and $B 2$ are schematic summaries of the projection pattern and neurotransmitter immunoreactivity of neurons projecting to the STG [data compiled from Nagy (1981); Kilman et al. (1999); Cazalets et al. (1990); Meyrand et al. (2000); this paper.] Red: FLRFamide-like immunoreactivity; blue: histamine-like immunoreactivity; green: GABA-like immunoreactivity; yellow: serotonin-like immunoreactivity; gray: either unknown or untested. 
Because the PS-equivalent neurons in other species were shown to express histaminergic phenotypes, we tested for the presence of this substance in PS neurons. Histamine immunocytochemical detection after retrograde dye migration from the stn performed in the embryo (Fig. 8A3) and the juvenile (Fig. 8A4) showed that PS neurons are histamine immunoreactive in the embryo (Fig. 8B3), juvenile (Fig. 8B4), and adult (data not shown). These data demonstrate that by contrast to GN1/2 neurons, PS neurons express all their known adult neurotransmitters early in development.

These data demonstrate (Fig. 9) that (1) projection neurons are present early in development but they do not necessarily express their adult neurotransmitter phenotype (e.g., GN1/2), and (2) neurons projecting to the same target network and expressing the same neuromodulator do not necessarily acquire this neuromodulator at the same ontogenetic time (compare PS and GN1/2).

\section{DISCUSSION}

Our results show that (1) all known neurons projecting to a neural network are present at developmental stage $65 \%$, (2) some ontogenetic changes take place in neurotransmitter alteration within the same set of projection neurons, and (3) during ontogeny, a given neuromodulatory substance does not synchronously appear in all neurons that will express it in the adult.

\section{Dye migration techniques}

To localize the somata of neurons projecting to the STG, we used retrograde dye migration. This technique has already been used in the crustacean STNS with different tracers such as Lucifer yellow (Cournil et al., 1990; Coleman et al., 1992; Nagy et al., 1994; Meyrand et al., 2000) and biocytin (Coleman et al., 1992). Both of these tracers have disadvantages. Biocytin is known to cross gap junctions, whereas Lucifer yellow migrates for only short distances and stains far fewer somata than other dyes (Coleman et al., 1992). Among available fluorescent dyes, we chose dextran tetramethyl rhodamine, which has a sufficiently high molecular weight (3000 MW) that it does not cross gap junctions (A. Mizrahi, personal communication). Moreover, this dye exhibits only weak photobleaching, which was an advantage in our studies combining dye migration and delayed immunocytochemical characterization of neurotransmitters. Using this dye, we found the same number of stained cells as described previously in anterior ganglia using different dyes (Cournil et al., 1991; Nagy et al., 1994; Meyrand et al., 2000).

Our data show some variability in the number of stained somata from one preparation to another at a given developmental stage. Such variability is inherent in the retrograde dye migration technique. For example, in the adult, the two PS somata, because of their characteristic position in the ivn, were always detected under transmitted light in the dissected STNS. However, after dye migration, the number of stained somata in the ivn ranged from 0 to 2 . This variability is not specific to this dye but has already been reported and discussed for other dyes in several species (Coleman et al., 1992; Nagy et al., 1994). Although variability was found in the embryo as well as adult, the maximal number of stained somata was identical when few somata were stained (two PS neurons; in the OG, three neurons stained through the on and two neurons stained through the son/ion; two neurons stained in the CoG through the ion) or was very close when more somata were stained (13 and 14 stained somata in the CoG through the son; 19 and 16 neurons stained in the CoG through both ion and son), and the mean and SD of stained somata were identical in all cases. Therefore, our data indicate that the population of central and peripheral projection neurons to the STG that are revealed by dye migration techniques is established at developmental stage $65 \%$. Furthermore, as performed previously by Coleman et al. (1992), we have counted the large fibers within the stn at different stages of development using electron microscopy techniques. This approach shows that the population of large fibers, previously described as modulatory fibers (Coleman et al., 1992), is quantitatively established early in development and corresponds to the number of central, peripheral, and STG neurons stained by dye migration from the stn toward the anterior ganglia or the STG and periphery (Y. Le Feuvre, V. S. Fenelon, B. Casasnovas, N. Mesmer-Dudons, and A. Alain, P. Meyrand, unpublished observations). Moreover, the basic projection scheme of these neurons is similar in the embryo (Fig. 9A2) and adult (Fig. 9B2). All neurons that could be identified in the adult after dye migration, on the basis of their soma morphology and projection pattern, could also be identified in the embryo using the same criteria.

\section{Early elaboration of projection neurons to motor networks}

Embryonic or larval motor networks may express rhythmic motor output long before receiving descending information that modulates the adult networks (Sillar et al., 1998; Branchereau et al., 2001). The acquisition of adult characteristics then depends on the establishment of functional descending inputs. In both vertebrates and invertebrates, neuromodulatory systems are composed of several subgroups of neurons (Harris-Warrick et al., 1992) or nuclei (ten Donkelaar, 2000) that are well defined in terms of anatomy and function. Numerous modulatory systems that modulate vertebrate motor networks have been shown to reach their targets at different developmental stages. Indeed, in all studied tetrapods, reticulospinal fibers reach the spinal cord first, followed by vestibulospinal fibers and, much later, by rubrospinal fibers (ten Donkelaar, 2000). In addition, within a given neuromodulatory system, axonal growth of modulatory fibers is generally assumed to carry neuromodulatory substances to target networks. Among neuromodulatory substances involved in the control of motor network activity, the ontogeny of 5-HT-containing fibers has been studied extensively. For example, the growth cones of raphe-spinal projection neurons are 5-HT immunopositive and progressively invade the spinal cord from the anterior to caudal part in Xenopus (van Mier et al., 1986). Similarly, mammalian raphe-spinal projections seem to display simultaneous growth and 5-HT expression (Rajaofetra et al., 1989; Kudo et al., 1993).

This ontogenetic work on the serotoninergic system has encouraged other studies on the timing of distinct projection systems using immunocytochemical detection of neuromodulatory substances. Using this approach on the STNS, we found that the motor target network progressively receives throughout development its adult complement of modulatory substances (Fenelon et al., 1998a, 1999; Kilman et al., 1999). However, in the present study, we show that all defined adult projection neurons seem to be present and reach their target network at mid-embryonic development, although at this time the adult complement of neurotransmitters is still lacking. Therefore, the progressive acquisition of neuromodulatory phenotypes may not necessarily depend on the ingrowth of projection neurons toward their target networks, but rather result from the acquisition of new neurotransmitter phenotypes within an already established set of projection fibers. However, although not yet investigated, axonal 
growth from the anterior ganglia to the target STG network may occur in very early development. Potentially, subsets of projection neurons that express their adult neurotransmitter phenotype at developmental stage $65 \%$ (for instance, the PS neurons) already expressed it during their axonal descent. By contrast, neurons such as GN1/2 reach their target network before their adult transmitters are expressed. Therefore, immunocytochemical data showing delayed acquisition of neuromodulatory substances (for instance, in spinal cord) do not necessarily imply that the fibers containing this substance were not projecting at earlier developmental stages. Indeed, such delayed acquisition of neurotransmitters has already been suggested in neurons of the ferret basal ganglia projecting to cortex (Henderson, 1991) as well as peripheral sensory neurons in the STNS of the lobster (Kilman et al., 1999).

\section{Phylogeny, ontogeny, and adult neuronal plasticity}

Our data indicate that the modulatory environment of target motor networks changes considerably during embryonic and larval development. Such plasticity contrasts with the stability observed in both the neuronal population that constitutes the target network (Fenelon et al., 1998a) and the organization of the modulatory system (this paper). This suggests that in the STNS, the major ontogenetic changes seem to be composed of the alteration of neurotransmitter expression within the same modulatory system, although channels or receptor expression may also be altered. Modulatory systems are responsible for dramatic alterations of the output of adult STG networks. Indeed, bath application of neuromodulatory substances (Harris-Warrick et al., 1992; Marder and Weimann, 1992; Blitz et al., 1995; Richards and Marder, 2000) or stimulation of identified modulatory neurons (Meyrand et al., 1991, 1994, 2000; Nagy et al., 1994; Norris et al., 1996; Blitz et al., 1999; Combes et al., 1999) elicits a wide variety of motor outputs from the same neuronal circuitry. Although the embryonic STNS generates a motor output different from the adult one (Casasnovas and Meyrand, 1995), it has been shown recently that embryo can generate adult-like activity patterns (Le Feuvre et al., 1999), suggesting that basic network architecture is similar in the embryo and adult. Moreover, as in the adult, the expression of embryonic circuitry depends strictly on the presence of projection neurons (Le Feuvre et al., 1999) and can be altered by neuromodulatory substances (Marder and Richards, 1999; Richards and Marder, 2000). Our results indicate that projection neurons are present in the embryo but that they do not express their adult neuromodulators. Therefore, the ontogenetic plasticity appears to result from changes in the neurotransmitters expressed within the preestablished neuronal motor system, rather than from drastic changes in the architecture of motor networks and of their modulatory input systems.

Interestingly, the basic organization of STG networks (Meyrand and Moulins, 1988a,b; Katz and Tazaki, 1992; Tazaki, 1993; Tazaki and Tazaki, 2000), as well as modulatory systems (Claiborne and Selverston, 1984; Cazalets et al., 1990; Katz and Tazaki, 1992; Coleman and Nusbaum, 1994; Nagy et al., 1994; Katz and Harris-Warrick, 1999; Meyrand et al., 2000), appears to be preserved across different species. It has been suggested that differences between species-specific motor output are attributable mainly to different control of CPGs by modulatory systems, which express different neurotransmitters for a given homologous input neuron (Meyrand et al., 2000). Therefore, differential control of a similar CPG by changes in neurotransmitter phenotype or differ- ential recruitment within similar modulatory inputs may account for ontogenetic, phylogenetic, and adult plasticity.

In vertebrates, adult CPGs can produce multiple motor outputs under the control of different modulatory signals (Sillar et al., 1997; Rossignol et al., 1998; Lieske et al., 2000). These CPGs are present very early in development (Sillar et al., 1997; Fenelon et al., 1998b; Kudo and Nishimaru, 1998; Branchereau et al., 2001) and also express multiple outputs in response to distinct modulatory environments (Sillar et al., 1998; Branchereau et al., 2001). The basic organization of CPGs for locomotion is highly conserved from lamprey to larval Xenopus to neonatal rats (Sillar et al., 1997). Furthermore, alteration in descending modulatory inputs across related species underlies the differential expression of species-specific motor activities (Woolston et al., 1994; Sillar et al., 1998). Together, this suggests that in both ontogeny and phylogeny, changes in the activity and neurotransmitter phenotype of descending inputs may produce multiple distinct modes of operation of the same CPG.

\section{REFERENCES}

Beltz B, Eisen JS, Flamm R, Harris-Warrick RM, Hooper SL, Marder E (1984) Serotonergic innervation and modulation of the stomatogastric ganglion of three decapod crustaceans (Panulirus interruptus, Homarus americanus and Cancer irroratus). J Exp Biol 109:35-54.

Birmingham JT, Szuts ZB, Abbott LF, Marder E (1999) Encoding of muscle movement on two time scales by a sensory neuron that switches between spiking and bursting modes. J Neurophysiol 82:2786-2797.

Blitz DM, Christie AE, Marder E, Nusbaum MP (1995) Distribution and effects of tachykinin-like peptides in the stomatogastric nervous system of the crab, Cancer borealis. J Comp Neurol 354:282-294.

Blitz DM, Christie AE, Coleman MJ, Norris BJ, Marder E, Nusbaum MP (1999) Different proctolin neurons elicit distinct motor patterns from a multifunctional neuronal network. J Neurosci 19:5449-5463.

Branchereau P, Morin D, Bonnot A, Ballion B, Chapron J, Viala D (2001) Development of lumbar rhythmic networks: from embryonic to neonate locomotor-like patterns in the mouse. Brain Res Bull, in press.

Cabana T, Martin GF (1984) Developmental sequence in the origin of descending spinal pathways. Studies using retrograde transport techniques in the North American opossum (Didelphis virginiana). Brain Res 317:247-263.

Casasnovas B, Meyrand P (1995) Functional differentiation of adult neural circuits from a single embryonic network. J Neurosci 15:5703-5718.

Cazalets JR, Nagy F, Moulins M (1990) Suppressive control of the crustacean pyloric network by a pair of identified interneurons. I. Modulation of the motor pattern. J Neurosci 10:448-457.

Claiborne BJ, Selverston AI (1984) Localization of stomatogastric IV neuron cell bodies in lobster brain. J Comp Physiol [A] 154:27-32.

Coleman MJ, Nusbaum MP (1994) Functional consequences of compartmentalization of synaptic input. J Neurosci 14:6544-6552.

Coleman MJ, Nusbaum MP, Cournil I, Claiborne BJ (1992) Distribution of modulatory inputs to the stomatogastric ganglion of the crab, Cancer borealis. J Comp Neurol 325:581-594.

Combes D, Meyrand P, Simmers J (1999) Motor pattern specification by dual descending pathways to a lobster rhythm-generating network. J Neurosci 19:3610-3619.

Commissiong JW (1983) Development of catecholaminergic nerves in the spinal cord of the rat. Brain Res 264:197-208.

Cournil I, Meyrand P, Moulins M (1990) Identification of all GABAimmunoreactive neurons projecting to the lobster stomatogastric ganglion. J Neurocytol 19:478-493.

Cournil I, Meyrand P, Moulins M (1991) A method for the determination of projection areas of GABA immunoreactive neurons in the invertebrate nervous system. J Neurosci Methods 39:53-63.

Cournil I, Casasnovas B, Helluy SM, Beltz BS (1995) Dopamine in the lobster Homarus gammarus: II. Dopamine-immunoreactive neurons and development of the nervous system. J Comp Neurol 362:1-16.

Fenelon VS, Casasnovas B, Faumont S, Meyrand P (1998a) Ontogenetic alteration in peptidergic expression within a stable neuronal population in lobster stomatogastric nervous system. J Comp Neurol 399:289-305.

Fenelon VS, Casasnovas B, Simmers J, Meyrand P (1998b) Development of rhythmic pattern generators. Curr Opin Neurobiol 8:705-709.

Fenelon VS, Kilman V, Meyrand P, Marder E (1999) Sequential developmental acquisition of neuromodulatory inputs to a central patterngenerating network. J Comp Neurol 408:335-351.

Harris-Warrick RM, Nagy F, Nusbaum MP (1992) Neuromodulation of stomatogastric networks by identified neurons and transmitters. In: Dynamic biological networks: the stomatogastric nervous system 
(Harris-Warrick RM, Marder E, Selverston AI, Moulins M, eds), pp 87-138. Cambridge, MA: MIT.

Helluy SM, Beltz BS (1991) Embryonic development of the American lobster (Homarus americanus): quantitative staging and characterization of an embryonic molt cycle. Biol Bull 180:355-371.

Henderson Z (1991) Early development of the nucleus basalis-cortical projection but late expression of its cholinergic function. Neuroscience 311-324.

Katz PS, Harris-Warrick RM (1989) Serotonergic/cholinergic muscle receptor cells in the crab stomatogastric nervous system. II. Rapid nicotinic and prolonged modulatory effects on neurons in the stomatogastric ganglion. J Neurophysiol 62:571-581.

Katz PS, Harris-Warrick RM (1999) The evolution of neuronal circuits underlying species-specific behavior. Curr Opin Neurobiol 9:628-633.

Katz PS, Tazaki K (1992) Comparative and evolutionary aspects of the crustacean stomatogastric system. In: Dynamic biological networks. The stomatogastric nervous system (Harris-Warrick RM, Marder E, Selverston AI, Moulins M, eds), pp 221-261. Cambridge, MA: MIT.

Katz PS, Eigg MH, Harris-Warrick RM (1989) Serotonergic/cholinergic muscle receptor cells in the crab stomatogastric nervous system. I. Identification and characterization of the gastropyloric receptor cells. J Neurophysiol 62:558-570.

Kilman V, Fenelon VS, Richards KS, Thirumalai V, Meyrand P, Marder E (1999) Sequential developmental acquisition of cotransmitters in identified sensory neurons of the stomatogastric nervous system of the lobsters, Homarus americanus and Homarus gammarus. J Comp Neurol 408:318-334.

Kudo N, Nishimaru H (1998) Reorganization of locomotor activity during development in the prenatal rat. Ann NY Acad Sci 860:306-317.

Kudo N, Furukawa F, Okado N (1993) Development of descending fibers to the rat embryonic spinal cord. Neurosci Res 16:131-141.

Le Feuvre Y, Fenelon VS, Meyrand P (1999) Central inputs mask multiple adult neural networks within a single embryonic network. Nature 402:660-664.

Lieske SP, Thoby-Brisson M, Telgkamp P, Ramirez JM (2000) Reconfiguration of the neural network controlling multiple breathing patterns: eupnea, sighs and gasps. Nat Neurosci 3:600-607.

Marder E, Calabrese RL (1996) Principles of rhythmic motor pattern generation. Physiol Rev 76:687-717.

Marder E, Richards KS (1999) Development of the peptidergic modulation of a rhythmic pattern generating network. Brain Res 848:35-44.

Marder E, Weimann JM (1992) Modulatory control of multiple task processing in the stomatogastric nervous system. In: Neurobiology of motor program selection (Kien J, McCrohan C, Winlow B, eds), pp 3-19. New York: Pergamon

McKinney RA, Capogna M, Durr R, Gahwiler BH, Thompson SM (1999) Miniature synaptic events maintain dendritic spines via AMPA receptor activation. Nat Neurosci 2:44-49.

Meyrand P, Moulins M (1988a) Phylogenetic plasticity of crustacean stomatogastric circuits. I. Extrinsic inputs to the pyloric circuit of the shrimp Palaemon serratus. J Exp Biol 138:133-153.

Meyrand P, Moulins M (1988b) Phylogenetic plasticity of crustacean stomatogastric circuits. I. Pyloric patterns and pyloric circuit of the shrimp Palaemon serratus. J Exp Biol 138:107-132.

Meyrand P, Simmers J, Moulins M (1991) Construction of a patterngenerating circuit with neurons of different networks. Nature 351:60-63.

Meyrand P, Simmers J, Moulins M (1994) Dynamic construction of a neural network from multiple pattern generators in the lobster stomatogastric nervous system. J Neurosci 14:630-644.

Meyrand P, Faumont S, Simmers J, Christie AE, Nusbaum MP (2000) Species-specific modulation of pattern-generating circuits. Eur J Neurosci 12:2585-2596.

Mulloney B, Hall WM (1991) Neurons with histaminelike immunoreactivity in the segmental and stomatogastric nervous systems of the crayfish Pacifastacus leniusculus and the lobster Homarus americanus. Cell Tissue Res 266:197-207.

Nagy F (1981) Etude de l'expression d'activités motrices rythmiques organisées par des générateurs paucineuroniques du système nerveux stomatogastrique des crustacés décapodes. Flexibilité intrinsèque aux réseaux moteurs; contrôle par les centres supérieurs; contrôle proprioceptif. PhD thesis, Université Bordeaux I.
Nagy F, Cardi P, Cournil I (1994) A rhythmic modulatory gating system in the stomatogastric nervous system of Homarus gammarus. I. Pyloricrelated neurons in the commissural ganglia. $J$ Neurophysiol 71:2477-2489.

Norris BJ, Coleman MJ, Nusbaum MP (1996) Pyloric motor pattern modification by a newly identified projection neuron in the crab stomatogastric nervous system. J Neurophysiol 75:97-108.

Okado N, Oppenheim RW (1985) The onset and development of descending pathways to the spinal cord in the chick embryo. J Comp Neurol 232:143-161.

Pindzola RR, Ho RH, Martin GF (1990) Development of catecholaminergic projections to the spinal cord in the North American opossum, Didelphis virginiana. J Comp Neurol 294:399-417.

Rajaofetra N, Sandillon F, Geffard M, Privat A (1989) Pre- and postnatal ontogeny of serotonergic projections to the rat spinal cord. J Neurosci Res 22:305-321.

Richards KS, Marder E (2000) The actions of crustacean cardioactive peptide on adult and developing stomatogastric ganglion motor patterns. J Neurobiol 44:31-44.

Rossignol S, Chau C, Brustein E, Giroux N, Bouyer L, Barbeau H, Reader TA (1998) Pharmacological activation and modulation of the central pattern generator for locomotion in the cat. Ann NY Acad Sci 860:346-359.

Sako H, Kojima T, Okado N (1986) Immunohistochemical study on the development of serotoninergic neurons in the chick: II. Distribution of cell bodies and fibers in the spinal cord. J Comp Neurol 253:79-91.

Senba E, Shiosaka S, Hara Y, Inagaki S, Sakanaka M, Takatsuki K, Kawai Y, Tohyama M (1982) Ontogeny of the peptidergic system in the rat spinal cord: immunohistochemical analysis. J Comp Neurol 208:54-66.

Sillar KT, Kiehn O, Kudo N (1997) Chemical modulation of vertebrate motor circuits. In: Neurons, networks, and motor behavior (Stein PG, Grillner S, Selverston AI, Stuart DG, eds), pp 183-193. Cambridge, MA: MIT.

Sillar KT, Reith C, McDearmid JR (1998) Development and aminergic neuromodulation of a spinal locomotor network controlling swimming in Xenopus larvae. Ann NY Acad Sci 860:318-332.

Simmers J, Moulins M (1988) Nonlinear interneuronal properties underlie integrative flexibility in a lobster disynaptic sensorimotor pathway. J Neurophysiol 59:757-777.

Skiebe P (1999) Allatostatin-like immunoreactivity in the stomatogastric nervous system and the pericardial organs of the crab Cancer pagurus, the lobster Homarus americanus, and the crayfish Cherax destructor and Procambarus clarkii. J Comp Neurol 403:85-105.

Tazaki K (1993) Motor pattern generation of the posterior cardiacplatepyloric system in the stomatogastric ganglion of the mantis shrimp Squilla oratoria. J Comp Physiol [A] 172:369-387.

Tazaki K, Tazaki Y (2000) Multiple motor patterns in the stomatogastric ganglion of the shrimp Penaeus japonicus. J Comp Physiol [A] 186:105-118.

ten Donkelaar HJ (2000) Development and regenerative capacity of descending supraspinal pathways in tetrapods: a comparative approach. Adv Anat Embryol Cell Biol 154:1-145.

Thoby-Brisson M, Simmers J (1998) Neuromodulatory inputs maintain expression of a lobster motor pattern-generating network in a modulation-dependent state: evidence from long-term decentralization in vitro. J Neurosci 18:2212-2225.

Trimmer BA, Kobierski LA, Kravitz EA (1987) Purification and characterization of FMRFamidelike immunoreactive substances from the lobster nervous system: isolation and sequence analysis of two closely related peptides. J Comp Neurol 266:16-26.

Turrigiano GG, Selverston AI (1991) Distribution of cholecystokininlike immunoreactivity within the stomatogastric nervous systems of four species of decapod crustacea. J Comp Neurol 305:164-176.

van Mier P, ten Donkelaar HJ (1984) Early development of descending pathways from the brain stem to the spinal cord in Xenopus laevis. Anat Embryol (Berl) 170:295-306.

van Mier P, Joosten HW, van Rheden R, ten Donkelaar HJ (1986) The development of serotonergic raphe spinal projections in Xenopus laevis. Int J Dev Neurosci 4:465-475.

Woolston AM, Wedderburn JF, Sillar KT (1994) Descending serotonergic spinal projections and modulation of locomotor rhythmicity in Rana temporaria embryos. Proc R Soc Lond B Biol Sci 255:73-79. 